TRANSACTIONS OF THE

AMERICAN MATHEMATICAL SOCIETY

Volume 301, Number 2, June 1987

\title{
STABILITY OF HARMONIC MAPS AND EIGENVALUES OF THE LAPLACIAN
}

\author{
HAJIME URAKAWA
}

Dedicated to Professor Ichiro Satake on his sixtieth birthday

\begin{abstract}
The index and nullity of the Hessian of the energy for every harmonic map are estimated above by a geometric quantity. The stability theory of harmonic maps is developed and as an application, the Kähler version of the LichnerowiczObata theorem about the first eigenvalue of the Laplacian is proved.
\end{abstract}

0. Introduction and statement of results. In this paper, we deal with the Hessian (the so-called Jacobi operator) of the energy for a harmonic map between two Riemannian manifolds, as the analogue of Morse theory for geodesics. The Morse theory for geodesics determines the homotopy type of the path space, using the notion of the index and nullity of the Hessian of the length of a geodesic. The celebrated Morse index theorem tells us that the index or nullity of a geodesic coincides with the number of conjugate points along the geodesic, and this gives an upper estimate for the index and nullity in terms of the length of the geodesic under a curvature assumption, giving a result known as the Morse-Schoenberg theorem:

TheOREM (MORSE - SCHOENBERG [G.K.M]). Assume that the sectional curvature ${ }^{N} K$ of a Riemannian manifold $(N, h)$ is bounded above by a positive constant ${ }^{N} K \leqslant a$. Then the nullity and index of a geodesic $\gamma ;[0,2 \pi] \rightarrow(N, h)$ satisfy

$$
\operatorname{Index}(\gamma)+\operatorname{Nullity}(\gamma) \leqslant(n-1)\left[L \frac{\sqrt{a}}{\pi}\right],
$$

where $L$ is the length of the geodesic $\gamma$ and $[x]$ is the integer part of a positive real number $x$.

Harmonic maps are the natural extensions of the notion of geodesic. They are defined as critical points of the energy on the space of smooth maps between two Riemannian manifolds (cf. [E.S, E.L]). Therefore it is reasonable to look for the analogue of Morse theory for harmonic maps. In order to do this we have to deal with the index and nullity of the Hessian of the energy and investigate their quantitative behavior for a general harmonic map.

Received by the editors October 30, 1985 and, in revised form, June 6, 1986. 1980 Mathematics Subject Classification. Primary 58E20; Secondary 58G11.

This work is supported by Max-Planck-Institut für Mathematik.

(C)1987 American Mathematical Society $0002-9947 / 87 \$ 1.00+\$ .25$ per page 
The first aim of this paper is to extend the above Morse-Schoenberg theorem to a general harmonic map, although we do not yet know the index theorem for harmonic maps. Namely we will give a general upper estimate on the index and nullity for every harmonic map in terms of a geometric quantity which coincides with the length in case of a geodesic (cf. Corollary 3.5). Note that the MorseSchoenberg theorem gives a stability theorem for a geodesic with a small length, i.e., the index and nullity vanish for a geodesic with a small length. Our upper estimate also gives a stability theorem (cf. Corollary 3.3) for a harmonic map with the above quantity small, which generalizes the stability theorem for a minimal immersion obtained by D. Hoffman [H], H. Mori [M], and S. Tanno [T2].

Now we call a harmonic map $\phi$ from a compact Riemannian manifold without boundary stable if the index of $\phi$ is zero (cf. [E.L]). We will study stable harmonic maps to determine what kind of harmonic maps are stable. Moreover, the second aim of this paper is to give an application of the stability of a harmonic map. It is well known that a holomorphic map between two compact Kähler manifolds is a stable harmonic map. In fact, it minimizes the energy within its homotopy class. In particular, the identity map of a compact Kähler manifold is stable. Using this fact, we give a Kähler version of Lichnerowicz-Obata's theorem about the lower estimate on the first eigenvalue of the Laplacian. More precisely we give

THEOREM 4.2. Let $(M, g)$ be a compact Kähler manifold whose Ricci curvature Ric $_{M}$ is bounded below by a positive constant $\alpha>0$. Then the first eigenvalue $\lambda_{1}(M, g)$ of the Laplacian satisfies $\lambda_{1}(M, g) \geqslant 2 \alpha$. If the equality holds, then $M$ admits a nonzero holomorphic vector field.

The third goal of this paper is to investigate a special kind of stable harmonic map. Y. L. Xin $[\mathbf{X}]$ gave a remarkable result that each nonconstant harmonic map from the canonical unit sphere $S^{n}, n \geqslant 3$, into another Riemannian manifold is instable. It is natural to ask the following:

Does there exist a deformation $g_{t}, 0<t<\infty$, of the standard metric $g_{1}$ on $S^{n}$ such that if $g_{t}$ is far from $g_{1}$, then $\left(S^{n}, g_{t}\right)$ admits a stable harmonic map?

In order to answer this question, we investigate the stability of the projection of the Riemannian submersion with totally geodesic fibers since the projection is a typical example of a harmonic map. Let $\phi ;(M, g) \rightarrow(N, h)$ be a Riemannian submersion with totally geodesic fibers. Following [B.B], we consider the canonical variation $g_{t}, 0<t<\infty$, of the metric $g$ which also gives a Riemannian submersion $\phi ;\left(M, g_{t}\right) \rightarrow(N, h)$ with totally geodesic fibers. Then we have:

THEOREM 7.3. Assume that the identity map of $(N, h)$ is stable. Then there exists a small number $\varepsilon$ such that for every $0<t<\varepsilon$, the Riemannian submersion $\phi ;\left(M, g_{t}\right)$ $\rightarrow(N, h)$ is stable.

Since the identity map of the complex projective space $\left(\mathbf{C} P^{n}, h\right)$ is stable, the Hopf fibering $\pi ;\left(S^{2 n+1}, g_{t}\right) \rightarrow\left(\mathbf{C} P^{n}, h\right)$ is stable for $0<t<\varepsilon$, for the canonical variation $g_{t}, 0<t<\infty$, with $g_{1}=$ canonical metric. This gives an example which contrasts with the instability theorem of Xin. 
Finally, we investigate homogeneous Riemannian submersions and calculate the index and nullity of the Hopf fibering (cf. Corollary 8.12).

ACKNOwLEDGment. This work was done during my stay in Bonn. I would like to express sincere gratitude to the Max-Planck-Institut für Mathematik for its hospitality.

\section{Chapter I. The INDEX AND NUllity OF A GENERAL HARMONIC MAP}

\section{Preliminaries.}

1.1. In this section, following [E.L], we describe the second variational formula of the energy functional obtained in [Ma, Sm].

Let $(M, g)$ and $(N, h)$ be Riemannian manifolds of dimension $m$ and $n$ respectively. Let $\phi ; M \rightarrow N$ be a smooth map. Let $E=\phi^{-1} T N$ be the bundle induced by $\phi$ over $M$ from the tangent bundle $T N$ of $N$. We denote by $\Gamma(E)$, the space of all sections $V$ of $E$, that is, $V \in \Gamma(E)$ implies that $V$ is a map of $M$ into $E$ such that $V_{x} \in T_{\phi(x)} N$ for all $x \in M$. For $X \in \Gamma(T M)$, we define $\phi_{*} X \in \Gamma(E)$ by $\left(\phi_{*} X\right)_{x}:=\phi_{*_{x}} X_{x} \in T_{\phi(x)} N, x \in M$, where $\phi_{* x}$ is the differential of $\phi$ at $x$. For $Y \in \Gamma(T N)$, we also define $\tilde{Y} \in \Gamma(E)$ by $\tilde{Y}_{x}:=Y_{\phi(x)}, x \in M$.

We denote by $\nabla$ and ${ }^{N} \nabla$ the Levi-Civita connections of $(M, g)$ and $(N, h)$ respectively. Then we give the induced connection $\tilde{\nabla}$ on $E$ by

$$
\tilde{\nabla}_{X} V:={ }^{N} \nabla_{\phi_{*} X} V
$$

for a tangent vector $X$ in $M$.

We define the tension field $\tau(\phi) \in \Gamma(E)$ of $\phi$ by

$$
\tau(\phi):=\sum_{i=1}^{m}\left(\tilde{\nabla}_{e_{i}} \phi_{*} e_{i}-\phi_{*} \nabla_{e_{i}} e_{i}\right),
$$

where $\left\{e_{i}\right\}_{i=1}^{m}$ is a (locally defined) orthonormal frame field on $M$. We call $\phi$ harmonic if $\tau(\phi)=0$. For a relatively compact domain $\Omega$ in $M$, the energy $E(\Omega, \phi)$ of $\phi$ on $\Omega$ is defined by

$$
E(\Omega, \phi):=\int_{\Omega} e(\phi)(x) * 1
$$

where $e(\phi)(x):=\frac{1}{2} \sum_{i=1}^{m} h\left(\phi_{*} e_{i}, \phi_{*} e_{i}\right)$ and $* 1$ is the volume element of $(M, g)$. We denote $E(\phi):=E(M, \phi)$ when defined. For an element $V$ in $\Gamma(E)$, let $\phi_{t} ; M \rightarrow N$ be a one-parameter family of maps from $M$ into $N$ with $\phi_{0}=\phi$, and $d \phi_{t}(x) /\left.d t\right|_{t=0}$ $=V_{x}, x \in M$. If $V \in \Gamma(E)$ has compact support, it is known (cf. [E.S, E.L, Ma]) that

$$
\left.\frac{d}{d t} E\left(\phi_{t}\right)\right|_{t=0}=-\int_{M} h(V, \tau(\phi)) * 1 .
$$

Moreover, if $\phi ;(M, g) \rightarrow(N, h)$ is harmonic and $V \in \Gamma(E)$ has compact support, then

$$
\left.\frac{d^{2}}{d t^{2}} E\left(\phi_{t}\right)\right|_{t=0}=\int_{M} h\left(V, J_{\phi} V\right) * 1
$$


where the operator $J_{\phi} ; \Gamma(E) \rightarrow \Gamma(E)$, called the Jacobi operator of $\phi$, is a second order elliptic differential operator given by

$$
J_{\phi} V:=-\sum_{i=1}^{m}\left\{\tilde{\nabla}_{e_{i}} \tilde{\nabla}_{e_{i}} V-\tilde{\nabla}_{\nabla_{e_{i}} e_{i}} V\right\}-\sum_{i=1}^{m}{ }^{N} R\left(\phi_{*} e_{i}, V\right) \phi_{*} e_{i},
$$

for $V \in \Gamma(E)$. Here ${ }^{N} R$ is the curvature tensor of $(N, h)$ given by

$$
{ }^{N} R(X, Y) Z:={ }^{N} \nabla_{[X, Y]} Z-{ }^{N} \nabla_{X}{ }^{N} \nabla_{Y} Z+{ }^{N} \nabla_{Y}{ }^{N} \nabla_{X} Z,
$$

for $X, Y, Z \in \Gamma(T N)$.

For a relatively compact domain $\Omega$ in $M$, let us consider the Dirichlet eigenvalue problem of $J_{\phi}$

$$
\begin{cases}J_{\phi} V=\lambda V & \text { on } \Omega \\ V=0 & \text { on } \partial \Omega .\end{cases}
$$

If $M$ is a closed manifold, we consider the eigenvalue problem of $J_{\phi}$

$$
J_{\phi} V=\lambda V, \quad V \in \Gamma(E) .
$$

It is known that the spectra of both problems (1.6) and (1.7) consist of discrete eigenvalues with finite multiplicities. The index of $\phi$ on $\Omega$, denoted by $\operatorname{Index}_{\Omega}(\phi)$, is defined as the sum of the multiplicities of the eigenvalues of the problem (1.6), and the index of $\phi$, denoted by Index $(\phi)$, is defined as the sum of the multiplicities of the eigenvalues of (1.7) when $M$ is a closed manifold. The dimension of the zero eigenspace of (1.6) (resp. (1.7)) is called the nullity of $\phi$ on $\Omega$ (resp. the nullity of $\phi$ ), denoted by $\operatorname{Nullity}_{\Omega}(\phi)$ (resp. Nullity $\left.(\phi)\right)$. The harmonic map $\phi ;(M, g) \rightarrow(N, h)$ is stable (resp. stable on $\Omega$ ) if Index $(\phi)=0$ (resp. $\left.\operatorname{Index}_{\Omega}(\phi)=0\right)$.

1.2. To estimate the index and nullity of a harmonic map, we introduce the quantity ${ }^{N} R^{\phi}$ or ${ }^{N} R_{\Omega}^{\phi}$ as follows.

DEFINITION 1.1. For a smooth map $\phi ;(M, g) \rightarrow(N, h)$, define ${ }^{N} R^{\phi}$ by

$$
{ }^{N} R^{\phi}:=\operatorname{Sup}_{x \in M} \operatorname{Sup}_{v \in T_{\phi(x)} N} \sum_{i=1}^{m} \frac{h\left({ }^{N} R\left(\phi_{*} e_{i}, v\right) \phi_{*} e_{i}, v\right)}{h(v, v)} .
$$

For a relatively compact domain $\Omega$ in $M$, define ${ }^{N} R_{\Omega}^{\phi}$ by

$$
{ }^{N} R_{\Omega}^{\phi}:=\operatorname{Sup}_{x \in \Omega} \operatorname{Sup}_{v \in T_{\phi(x)}} \sum_{i=1}^{m} \frac{h\left({ }^{N} R\left(\phi_{*} e_{i}, v\right) \phi_{*} e_{i}, v\right)}{h(v, v)} .
$$

Note that these quantities do not depend on the choice of $\left\{e_{i}\right\}_{i=1}^{m}$. We have immediately

LEMMA 1.2. Assume that the sectional curvature ${ }^{N} K$ of $(N, h)$ is bounded above by a positive constant a so that ${ }^{N} K(\pi) \leqslant$ a for all planes $\pi$ in $T_{y} N, y \in N$. Then we have

$$
\begin{gathered}
{ }^{N} R^{\phi} \leqslant 2 a E^{\infty}(\phi), \\
{ }^{N} R_{\Omega}^{\phi} \leqslant 2 a E^{\infty}(\Omega, \phi) .
\end{gathered}
$$

Here $E^{\infty}(\phi):=\sup _{x \in M} e(\phi)(x)$ and $E^{\infty}(\Omega, \phi):=\operatorname{Sup}_{x \in \Omega} e(\phi)(x)$. 
In fact, it is obvious that

$$
\sum_{i=1}^{m} h\left({ }^{N} R\left(\phi_{*} e_{i}, v\right) \phi_{*} e_{i}, v\right) \leqslant a\left\{\sum_{i=1}^{m} h\left(\phi_{*} e_{i}, \phi_{*} e_{i}\right)\right\} h(v, v)
$$

at each point of $M$. Note that

$$
E(\Omega, \phi) \leqslant E^{\infty}(\Omega, \phi) \operatorname{Vol} \Omega \text { and } E(\phi) \leqslant E^{\infty}(\phi) \operatorname{Vol} M \quad \text { if } \operatorname{Vol} M<\infty .
$$

EXAmPle 1.3. Let $\phi ;(M, g) \rightarrow(N, h)$ be an isometric immersion. Then $e(\phi)(x)$ $=m / 2$ at each point. Therefore

$$
\begin{gathered}
E^{\infty}(\phi)=E^{\infty}(\Omega, \phi)=m / 2, \\
{ }^{N} R_{\Omega}^{\phi} \leqslant{ }^{N} R^{\phi} \leqslant m a,
\end{gathered}
$$

for every relatively compact domain $\Omega$ in $M$. In particular, let $\phi ;[0,2 \pi] \rightarrow(N, h)$ be a geodesic with length $L$. Then

$$
E^{\infty}(\phi)=L^{2} / 8 \pi^{2}
$$

EXAmple 1.4. Let $\phi ;(M, g) \rightarrow(N, h)$ be a Riemannian submersion (cf. $§ 6)$. Then we can choose an orthonormal local frame $\left\{e_{i}\right\}_{i=1}^{m}$ on $M$ such that $\phi_{*} e_{i}=e_{i}^{\prime}$, $1 \leqslant i \leqslant n$, and $\phi_{*} e_{i}=0, n+1 \leqslant i \leqslant m$, where $m=\operatorname{dim} M, n=\operatorname{dim} N$, and $\left\{e_{i}^{\prime}\right\}_{i=1}^{n}$ is an orthonormal local frame on $N$. Then the Ricci curvature of $(N, h)$, $\operatorname{Ric}_{N}(v), v \in T_{\phi(x)} N$, is by definition $\sum_{i=1}^{m} h\left({ }^{N} R\left(\phi_{*} e_{i}, v\right) \phi_{*} e_{i}, v\right) / h(v, v)$. Therefore, since $\phi$ is surjective, we have

$$
{ }^{N} R^{\phi}=\operatorname{Sup}_{N} \operatorname{Ric}_{N} \quad \text { and } \quad{ }^{N} R_{\Omega}^{\phi}=\operatorname{Sup}_{\phi(\Omega)} \operatorname{Ric}_{N} .
$$

2. An estimate for the index and nullity of a harmonic map. At first, let us recall a method of Berard and Gallot (cf. [B.G]) to give estimates of the Betti number and dimension of the moduli space of Einstein metrics, and the dimension of harmonic spinors. In this section, we point out that their method works well in the case of the Dirichlet eigenvalue problem for a relatively compact domain $\Omega$ in a complete Riemannian manifold $(M, g)$.

Let $(M, g)$ be a complete Riemannian manifold of dimension $m$, and $E$, a vector bundle over $M$ with an inner product $\langle\cdot, \cdot\rangle$ and a connection $\tilde{\nabla}$ compatible with $\langle\cdot, \cdot\rangle$, that is,

$$
\nabla_{X}\left\langle s, s^{\prime}\right\rangle=\left\langle\tilde{\nabla}_{X} s, s^{\prime}\right\rangle+\left\langle s, \tilde{\nabla}_{X} s^{\prime}\right\rangle, \quad X \in \Gamma(T M), s, s^{\prime} \in \Gamma(E) .
$$

Then we can define the rough Laplacian $\bar{\Delta}$ on $E$ so that

$$
\bar{\Delta} s:=\sum_{i=1}^{m}\left\{\tilde{\nabla}_{e_{i}} \tilde{\nabla}_{e_{i}} s-\tilde{\nabla}_{\nabla_{e_{i}} e_{i}} s\right\}, \quad s \in \Gamma(E),
$$

where $\left\{e_{i}\right\}_{i=1}^{m}$ is an orthonormal local frame field on $M$.

Let $\bar{\lambda}_{1}(\Omega) \leqslant \bar{\lambda}_{2}(\Omega) \leqslant \cdots \leqslant \bar{\lambda}_{i}(\Omega) \leqslant \cdots$ be the spectrum of the Dirichlet eigenvalue problem of the rough Laplacian $\bar{\Delta}(2.1)$ of the vector bundle $E$ over a relatively compact domain $\Omega$ in $M$ :

$$
\begin{cases}-\bar{\Delta} s=\lambda s & \text { on } \Omega \\ s=0 & \text { on } \partial \Omega\end{cases}
$$


where $s$ is a section of $E$ on the closure $\bar{\Omega}$ of $\Omega$. Consider the zeta function $\bar{Z}_{E, \Omega}(t)$ defined by

$$
\bar{Z}_{E, \Omega}(t):=\sum_{i=1}^{\infty} e^{-t \bar{\lambda}_{i}(\Omega)}, \quad t>0 .
$$

Similarly, let $\lambda_{1}(\Omega) \leqslant \lambda_{2}(\Omega) \leqslant \cdots \leqslant \lambda_{i}(\Omega) \leqslant \cdots$ be the spectrum of the Dirichlet eigenvalue problem of the Laplace-Beltrami operator $\Delta_{M}$ for the domain $\Omega$, anc $Z_{\Omega}(t)$ be the zeta function defined by

$$
Z_{\Omega}(t):=\sum_{i=1}^{\infty} e^{-t \lambda_{i}(\Omega)}, \quad t>0 .
$$

Then we have the analogue of a theorem of Hess, Schrader and Uhlenbrock:

THEOREM 2.1. If $l$ is the rank of $E$ then

$$
\bar{Z}_{E, \Omega}(t) \leqslant l Z_{\Omega}(t), \quad t>0 .
$$

Proof. This can be proved as in [B.G]. Assume that $s(t, x) \in E_{x}, t>0, x \in \bar{\Omega}$, satisfies the heat equation with the Dirichlet boundary condition

$$
\begin{cases}\left(\frac{\partial}{\partial t}-\bar{\Delta}\right) s(t, x)=0 & \text { on }(0, \infty) \times \Omega \\ s(t, x)=0 & \text { on }(0, \infty) \times \partial \Omega\end{cases}
$$

For each $\varepsilon>0$, let $f_{\varepsilon}:=\left(|s|^{2}+\varepsilon^{2}\right)^{1 / 2}$ on $(0, \infty) \times \bar{\Omega}$. Then it can be proved as in [H.S.U.] that $\langle-\bar{\Delta} s, s\rangle \leqslant f_{\varepsilon}\left(-\Delta_{M} f_{\varepsilon}\right)$ on $(0, \infty) \times \Omega$. Therefore $f_{\varepsilon}$ satisfies

$$
\left(\frac{\partial}{\partial t}-\Delta_{M}\right) f_{\varepsilon} \leqslant 0 \quad \text { on }(0, \infty) \times \Omega
$$

Then we can apply $f_{\varepsilon}$ to the following maximum principle for the heat kernel:

Theorem (MAXimum Principle). Let $\Omega$ be a relatively compact domain in $M$, and let $0<T<\infty$. Assume that $u$ is a real valued continuous function on $[0, T] \times \Omega$ and satisfies the inequality

$$
\frac{\partial}{\partial t} u-\Delta_{M} u \leqslant 0 \quad \text { on }(0, T) \times \Omega
$$

Then $u$ attains its maximum on the set $\{0\} \times \Omega$ or $[0, T] \times \partial \Omega$.

For proof, see [F, p. 204].

If $f_{\varepsilon}(0, x) \leqslant f(0, x)+\varepsilon$, then $f_{\varepsilon}(t, x) \leqslant f(t, x)+\varepsilon$. Hence for every integrable section $s$ of $E$ on $\bar{\Omega}$ with the Dirichlet condition $s=0$ on $\partial \Omega$, we have

$$
\left|\left(e^{t \bar{\Delta}_{S}}\right)(x)\right| \leqslant\left(e^{t \Delta_{M}}|s|\right)(x) .
$$

Therefore applying $s(z)=\sum_{i=1}^{l} \delta_{z, y} u_{j}(z)$ to (2.4), where $\delta_{z, y}$ is the Dirac function at $y$ and $\left\{u_{j}(z)\right\}_{j=1}^{l}$ is an orthonormal basis of the fiber $E_{z}$ at each point $z$ in $M$, and noting that $|s(z)|=l \delta_{z, y}$, we have the desired inequality (2.3). Q.E.D.

We denote the spectrum of the Dirichlet eigenvalue problem of $J_{\phi}$ on $\Omega$ by

$$
\tilde{\lambda}_{1}(\Omega) \leqslant \tilde{\lambda}_{2}(\Omega) \leqslant \cdots \leqslant \tilde{\lambda}_{i}(\Omega) \leqslant \cdots
$$


and define $\tilde{Z}_{\Omega}(t):=\sum_{i=1}^{\infty} e^{-t \tilde{\lambda}_{i}(\Omega)}$. Then we have

Proposition 2.2. Let $\Omega$ be a relatively compact domain in a complete Riemannian manifold $(M, g)$. Let $\phi ;(M, g) \rightarrow(N, h)$ be a harmonic map. Then

$$
\operatorname{Index}_{\Omega}(\phi)+\operatorname{Nullity}_{\Omega}(\phi) \leqslant \tilde{Z}_{\Omega}(t) \leqslant n \operatorname{Inf}\left\{e^{t^{N_{R}}{ }_{\Omega}} Z_{\Omega}(t) ; 0<t<\infty\right\},
$$

where $n=\operatorname{dim} N,{ }^{N} R_{\Omega}^{\phi}$ is defined in $\S 1$, and $Z_{\Omega}(t)$ is the zeta function of the Dirichlet eigenvalue of $\Delta_{M}$ on $\Omega$ defined by (2.2).

\section{The index and the nullity of a harmonic map from a domain.}

3.1. We retain the notation of $\S 2$. We have

THEOREM 3.1. Let $\Omega$ be a relatively compact domain in a complete Riemannian manifold $(M, g)$, with $\phi ;(M, g) \rightarrow(N, h)$ a harmonic map of $(M, g)$ into an arbitrary Riemannian manifold $(N, h)$ of dimension $n$. Then

(i) $\lambda_{1}(\Omega) \geqslant{ }^{N} R_{\Omega}^{\phi} \Rightarrow \operatorname{Index}_{\Omega}(\phi)=0$ and $\operatorname{Nullity}_{\Omega}(\phi) \leqslant n$,

(ii) $\lambda_{1}(\Omega)>{ }^{N} R_{\Omega}^{\phi} \Rightarrow \operatorname{Index}_{\Omega}(\phi)=\operatorname{Nullity}_{\Omega}(\phi)=0$.

That is, if $\lambda_{1}(\Omega) \geqslant{ }^{N} R_{\Omega}^{\phi}$, then the harmonic map $\phi ;(M, g) \rightarrow(N, h)$ is stable on $\Omega$.

Proof. By Proposition 2.2, the zeta function $\tilde{Z}_{\Omega}(t)=\sum_{=1}^{\infty} e^{-t \tilde{\lambda}_{i}(\Omega)}$ of $J_{\phi}$ on $\Omega$ satisfies

$$
\tilde{Z}_{\Omega}(t) \leqslant n e^{t{ }^{N} R_{\Omega}^{\phi}} Z_{\Omega}(t)=n e^{t\left({ }^{N} R_{\Omega}^{\phi}-\lambda_{1}(\Omega)\right)}\left\{1+\sum_{i=2}^{\infty} e^{t\left(\lambda_{1}(\Omega)-\lambda_{i}(\Omega)\right)}\right\},
$$

where $\lambda_{1}(\Omega) \leqslant \lambda_{2}(\Omega) \leqslant \cdots \leqslant \lambda_{i}(\Omega) \leqslant \cdots$ is the spectrum of the Dirichlet eigenvalue problem of the Laplace-Beltrami operator $\Delta_{M}$ on $\Omega$. Noting the fact that $\lambda_{i}(\Omega)>\lambda_{1}(\Omega), i=2,3, \ldots$, the assumption ${ }^{N} R_{\Omega}^{\phi} \leqslant \lambda_{1}(\Omega)$ implies that the limit of the right-hand side of the above inequality is less than or equal to $n$ when $t \rightarrow \infty$. Then Index ${ }_{\Omega}(\phi)=0$ and Nullity $_{\Omega}(\phi) \leqslant n$. If ${ }^{N} R_{\Omega}^{\phi}<\lambda_{1}(\Omega)$, the limit of the righthand side of the inequality is zero when $t \rightarrow \infty$. Therefore $\operatorname{Index}_{\Omega}(\phi)=\operatorname{Nullity}_{\Omega}(\phi)$ $=0$. Q.E.D.

COROLlaRY 3.2. Let $B_{r}(0)$ be a geodesic ball with radius $r$ whose center is a certain point $o$ in the $m$-dimensional standard unit sphere $\left(S^{m}\right.$, can) of constant curvature one. We choose the radius $r$ with $0<r<\pi / 2$ in such a way that $\lambda_{1}\left(B_{r}(0)\right)=m-1$. Then, for every domain $\Omega$ in $S^{m}$ whose volume $\operatorname{Vol}(\Omega)$ is less than or equal to the volume $\operatorname{Vol}\left(B_{r}(0)\right)$, the identity map id; $\left(S^{m}, \mathrm{can}\right) \rightarrow\left(S^{m}, \mathrm{can}\right)$ is stable on $\Omega$.

Proof. By Example 1.4, we have ${ }^{N} R_{\Omega}^{\phi}=m-1$ for every domain $\Omega$ in $S^{m}$. In this case, Theorem 3.1 implies that, if $\lambda_{1}(\Omega) \geqslant m-1$, then the identity map $\phi=\mathrm{id}$; $\left(S^{m}\right.$, can $) \rightarrow\left(S^{m}\right.$, can) is stable on $\Omega$. By a theorem of P. Bérard and D. Meyer (cf. [B.M] $)$, if $\operatorname{Vol}(\Omega) \leqslant \operatorname{Vol}\left(B_{r}(0)\right)$, then $\lambda_{1}(\Omega) \geqslant \lambda_{1}\left(B_{r}(0)\right)=m-1$. Q.E.D.

It is known (cf. [C.L, B.G, U2]) that there exists a positive constant $C(M, g)$ depending only on $(M, g)$ such that the eigenvalues $\lambda_{i}(\Omega)$ of the Dirichlet eigenvalue problem of the Laplace-Beltrami operator $\Delta_{M}$ on the domain $\Omega$ satisfy

$$
\lambda_{i}(\Omega) \geqslant C(M, g) \operatorname{Vol}(\Omega)^{-2 / m} i^{2 / m}, \quad i=1,2, \ldots,
$$


where $m=\operatorname{dim} M$. In particular,

$$
\lambda_{1}(\Omega) \geqslant C(M, g) \operatorname{Vol}(\Omega)^{-2 / m}
$$

Thus Theorem 3.1 implies

COROllary 3.3. Let $\Omega$ be a relatively compact domain in a complete Riemannian manifold $(M, g)$, with $\phi ;(M, g) \rightarrow(N, h)$ a harmonic map. Then

$$
C(M, g) \operatorname{Vol}(\Omega)^{-2 / m} \geqslant{ }^{N} R_{\Omega}^{\phi} \Rightarrow \phi \text { is stable on } \Omega \text {. }
$$

In particular, assume that the sectional curvature ${ }^{N} K$ of $(N, h)$ is bounded above by a positive constant ${ }^{N} K \leqslant a$. Then

$$
C(M, g) \operatorname{Vol}(\Omega)^{-2 / m} \geqslant 2 a E^{\infty}(\Omega, \phi) \Rightarrow \phi \text { is stable on } \Omega .
$$

If $\Omega$ is "small" in $(M, g)$, then $\operatorname{Vol}(\Omega)^{-2 / m}$ tends to infinity and ${ }^{N} R_{\Omega}^{\phi}$ still. remains bounded. Therefore Corollary 3.3 implies that a harmonic map $\phi ;(M, g)$ $\rightarrow(N, h)$ is stable on a "sufficiently small" domain $\Omega$ in $M$.

3.2. In this section, we estimate $\operatorname{Index}_{\Omega}(\phi)$ and $\operatorname{Nullity}_{\Omega}(\phi)$. By Proposition 2.2 and (3.1), we have

$$
\begin{aligned}
\operatorname{Index}_{\Omega}(\phi)+\operatorname{Nullity}_{\Omega}(\phi) & \leqslant n \operatorname{Inf}\left\{e^{t^{N} R_{\Omega}^{\phi}} Z_{\Omega}(t) ; 0<t<\infty\right\} \\
& \leqslant n \operatorname{Inf}\left\{e^{t^{N} R_{\Omega}^{\phi}} \sum_{k=1}^{\infty} e^{-t C(M, g) \operatorname{Vol}(\Omega)^{-2 / m} k^{2 / m}} ; 0<t<\infty\right\} \\
& \leqslant n \operatorname{Inf}\left\{e^{a t / b} \sum_{k=1}^{\infty} e^{-t k^{2 / m}} ; 0<t<\infty\right\},
\end{aligned}
$$

where we put $m=\operatorname{dim} M, n=\operatorname{dim} N, a:={ }^{N} R_{3}^{\phi}$, and $b:=C(M, g) \operatorname{Vol}(\Omega)^{-2 / m}$. In case $a \leqslant b$, we have Corollary 3.3. So assume $a>b$. We put $a / b=1+D, D>0$. We may write

$$
e^{a t / b} \sum_{k=1}^{\infty} e^{-t k^{2 / m}}=e^{(a / b-1) t} \sum_{k=1}^{\infty} e^{-\left(k^{2 / m}-1\right) t}
$$

In case $m=1,2$, the right-hand side of (3.3)

$$
\leqslant e^{(a / b-1) t} \sum_{k=0}^{\infty} e^{-t k}=e^{(a / b-1) t}\left(1-e^{-t}\right)^{-1} .
$$

Putting $e^{t}=1+1 / D$, we have

$$
\operatorname{Inf}\left\{e^{a t / b} \sum_{k=1}^{\infty} e^{-t k^{2 / m}} ; 0<t<\infty\right\} \leqslant\left(1+\frac{1}{D}\right)^{D}(1+D) .
$$


In case $m \geqslant 3$,

$$
\begin{aligned}
\sum_{k=1}^{\infty} e^{-t\left(k^{2 / m}-1\right)} & =1+e^{t} \sum_{k=2}^{\infty} e^{-t k^{2 / m}} \\
& \leqslant 1+e^{t} \int_{1}^{\infty} e^{-t x^{2 / m}} d x \\
& =1+\frac{m}{2} t^{-m / 2} \int_{t}^{\infty} z^{m / 2-1} e^{-z} d z \\
& \leqslant\left\{\begin{array}{l}
1+\frac{m}{2} t^{-m / 2} p ! e^{-t} \sum_{k=0}^{p} \frac{t^{k}}{k !}, \quad \text { if } m=2(p+1), p \geqslant 1, \\
1+\frac{m}{2} t^{-(m+1) / 2} p ! e^{-t} \sum_{k=0}^{p} \frac{t^{k}}{k !}, \quad \text { if } m=2 p+1, p \geqslant 1 .
\end{array}\right.
\end{aligned}
$$

Putting $e^{t}=1+1 / D$, we have

$$
\begin{aligned}
& \operatorname{Inf}\left\{e^{a t / b} \sum_{k=1}^{\infty} e^{\left.-t k^{2 / m} ; 0<t<\infty\right\}}\right. \\
& \qquad \begin{cases}\left(1+\frac{1}{D}\right)^{D}\{1+P(D)\}, & \text { if } m=2(p+1), p \geqslant 1, \\
\left(1+\frac{1}{D}\right)^{D}\{1+Q(D)\}, & \text { if } m=2 p+1, p \geqslant 1,\end{cases}
\end{aligned}
$$

where

$$
\begin{aligned}
& P(D):=(p+1) ! \sum_{k=0}^{p} \frac{1}{k !}\left\{\frac{1}{\log \left(1+\frac{1}{D}\right)}\right\}^{p+1-k}, \quad \text { if } m=2(p+1), p \geqslant 1 \\
& Q(D):=\frac{m}{2} p ! \sum_{k=0}^{p} \frac{1}{k !}\left\{\frac{1}{\log \left(1+\frac{1}{D}\right)}\right\}^{p+1-k}, \quad \text { if } m=2 p+1, p \geqslant 1 .
\end{aligned}
$$

We can give another estimate of $\operatorname{Index}_{\Omega}(\phi)$ and $\operatorname{Nullity}_{\Omega}(\phi)$. In fact, we have

$$
\sum_{k=1}^{\infty} e^{-t k^{2 / m}} \leqslant \int_{0}^{\infty} e^{-t x^{2 / m}} d x=\Gamma\left(\frac{m}{2}+1\right) t^{-m / 2}
$$

Therefore we obtain

$$
\operatorname{Inf}\left\{e^{a t / b} \sum_{k=1}^{\infty} e^{-t k^{2 / m}} ; 0<t<\infty\right\} \leqslant \frac{\Gamma(m / 2+1) e^{m / 2}}{(m / 2)^{m / 2}}\left(\frac{a}{b}\right)^{m / 2} .
$$

Summing up, we obtain

THEOREM 3.4. Let $\Omega$ be a relatively compact domain in a complete Riemannian manifold $(M, g)$, and $\phi ;(M, g) \rightarrow(N, h)$, a harmonic map. Then $\operatorname{Index}_{\Omega}(\phi)$ and Nullity $_{\Omega}(\phi)$ are estimated in terms of the quantity $D:={ }^{N} R_{\Omega}^{\phi} C(M, g)^{-1} \operatorname{Vol}(\Omega)^{2 / m}-1$ as follows:

(i) For $m=1,2$,

$$
\operatorname{Index}_{\Omega}(\phi)+\operatorname{Nullity}_{\Omega}(\phi) \leqslant n(1+1 / D)^{D}\{1+D\} .
$$

(ii) For $m=2(p+1), p \geqslant 1$,

$$
\operatorname{Index}_{\Omega}(\phi)+\operatorname{Nullity}_{\Omega}(\phi) \leqslant n(1+1 / D)^{D}\{1+P(D)\} .
$$


(iii) For $m=2 p+1, p \geqslant 1$,

$$
\operatorname{Index}_{\Omega}(\phi)+\operatorname{Nullity}_{\Omega}(\phi) \leqslant n(1+1 / D)^{D}\{1+Q(D)\} \text {. }
$$

(iv) For $m \geqslant 1$,

$$
\operatorname{Index}_{\Omega}(\phi)+\operatorname{Nullity}_{\Omega}(\phi) \leqslant n \frac{\Gamma(m / 2+1) e^{m / 2}}{(m / 2)^{m / 2}}(1+D)^{m / 2},
$$

where $P(D)$ and $Q(D)$ are the functions of $D$ given by (3.4), (3.5), respectively, and $m=\operatorname{dim} M, n=\operatorname{dim} N$.

REMARK. Since the function

$$
f(D)=\frac{1}{\log (1+1 / D)}
$$

of $D$ satisfies $f(D) \rightarrow 0$ as $D \rightarrow 0$ and $f(D) \sim D$ as $D \rightarrow \infty$, the functions $P(D)$ and $Q(D)$ satisfy $\lim _{D \rightarrow 0} P(D)=\lim _{D \rightarrow 0} Q(D)=0$, and $P(D) \sim(m / 2) ! D^{m / 2}$, and $Q(D) \sim(m / 2)((m-1) / 2) ! D^{(m+1) / 2}$ as $D \rightarrow \infty$.

Using (1.11), we obtain

Corollary 3.5. Assume that the sectional curvature ${ }^{N} K$ of $(N, h)$ is bounded above by a positive constant $a$. Let $\Omega$ be a relatively compact domain in a complete Riemannian manifold $(M, g)$, and $\phi ;(M, g) \rightarrow(N, h)$ a harmonic map. Then

$\operatorname{Index}_{\Omega}(\phi)+\operatorname{Nullity}_{\Omega}(\phi) \leqslant n \Gamma\left(\frac{m}{2}+1\right)\left\{\frac{C(M, g)^{-1} e a}{m}\right\}^{m / 2} E^{\infty}(\Omega, \phi)^{m / 2} \operatorname{Vol}(\Omega)$.

In particular, in the case $(M, g)=\left(\mathbf{R}^{m}\right.$, can $)$, the standard Euclidean space, since $C\left(\mathbf{R}^{m}\right.$, can $)=4 \pi^{2} \omega_{m}^{-2 / m}(c f .[\mathbf{U} 2])$ with $\omega_{m}=\pi^{m / 2} / \Gamma(m / 2+1)$, the volume of the unit ball, we have, for every harmonic map $\phi ;\left(\mathbf{R}^{m}\right.$, can $) \supset \Omega \rightarrow(N, h)$,

$$
\operatorname{Index}_{\Omega}(\phi)+\operatorname{Nullity}_{\Omega}(\phi) \leqslant n\left(\frac{e a}{m \pi}\right)^{m / 2} E^{\infty}(\Omega, \phi)^{m / 2} \operatorname{Vol}(\Omega)
$$

REMARK. It seems that the index and nullity of harmonic maps might be estimated above in terms of the quantity $\int_{\Omega} e(\phi)^{m / 2} * 1$.

In the case of a closed manifold $M$, we get the following

THEOREM 3.6. Let $(M, g)$ be a closed Riemannian manifold of dimension $m \geqslant 2$ whose Ricci curvature $\mathrm{Ric}_{M}$ is bounded below by a positive constant $(m-1) \delta>0$. Let $\phi ;(M, g) \rightarrow(N, h)$ be a harmonic map of $(M, g)$ into an arbitrary Riemannian manifold $(N, h)$ of dimension $n$.

(i) In case $m \geqslant 3$,

$$
\operatorname{Index}(\phi)+\operatorname{Nullity}(\phi) \leqslant n\left(1+\frac{1}{A}\right)^{A}\left\{1+(m-1) ! m^{m-1} A(1+A)^{m-1}\right\},
$$

where $A:={ }^{N} R^{\phi} / m \delta$ and ${ }^{N} R^{\phi}$ is the quality in $\S 1$.

(ii) In case $m=2$,

$$
\operatorname{Index}(\phi)+\operatorname{Nullity}(\phi) \leqslant n(1+1 / B)^{B}\left\{1+4 B^{2}\right\},
$$

where $B:={ }^{N} R^{\phi} / \delta$.

The proof is omitted. 


\section{Chapter II. Stability OF THE IDENTITy MAP}

4. A Kähler version of the Lichnérowicz-Obata theorem. In this chapter, we deal with the Jacobi operator of the identity map. Let $(M, g)$ be a closed Riemannian manifold of dimension $m$. The identity map id ${ }_{M} ;(M, g) \rightarrow(M, g)$ of $(M, g)$ is harmonic (cf. [E.S]), and the Riemannian manifold $(M, g)$ is stable (cf. [Na]) if the identity map $\mathrm{id}_{M}$ is stable. The corresponding Jacobi operator $J:=J_{\mathrm{id}_{M}}$ is a differential operator acting on the space $\Gamma(T M)$ of all vector fields on $M$ given by

$$
J V=-\sum_{i=1}^{m}\left(\nabla_{e_{i}} \nabla_{e_{i}} V-\nabla_{\nabla_{e_{i}} e_{i}} V\right)-\rho(V), \quad V \in \Gamma(T M),
$$

where $\nabla$ is the Levi-Civita connection of $(M, g), \rho(V):=\sum_{i=1}^{m} R\left(e_{i}, V\right) e_{i}$, and

$$
\rho(U, V):=g(\rho(U), V)=\sum_{i=1}^{m} g\left(R\left(e_{i}, U\right) e_{i}, V\right)
$$

is the Ricci tensor (cf. [Ma, Sm]). Under the identification of $T M$ with $T^{*} M$ with respect to the metric $g$, the Hodge Laplacian $\Delta=d \delta+\delta d$ on $\Gamma\left(T^{*} M\right)$ induces a differential operator, denoted by the same letter and called also as the Hodge Laplacian, on $\Gamma(T M)$, where $\delta$ is the codifferential operator of $d$ with respect to the metric $g$ on $M$. Then the Weitzenböck formula for the Hodge operator $\Delta$ tells us that

$$
\Delta V=-\sum_{i=1}^{m}\left(\nabla_{e_{i}} \nabla_{e_{i}} V-\nabla_{\nabla_{e_{i}} e_{i}} V\right)+\rho(V), \quad V \in \Gamma(T M),
$$

so that

$$
J=\Delta-2 \rho .
$$

Then we have immediately

LEMMA 4.1. Let $\lambda_{1}^{1}(M)\left(\right.$ resp. $\left.\lambda_{1}(M)\right)$ be the first (resp. first nonzero) eigenvalue of the Hodge Laplacian (resp. the Laplace-Beltrami operator $\Delta_{M}$ ) on 1-forms (resp. smooth functions) on $M$. Then

(i) $(M, g)$ is stable $\Rightarrow 2 \operatorname{Inf}_{\operatorname{Ric}_{M}} \leqslant \lambda_{1}^{1}(M) \leqslant \lambda_{1}(M)$,

(ii) $\lambda_{1}^{1}(M) \geqslant 2 \operatorname{Sup} \operatorname{Ric}_{M} \Rightarrow(M, g)$ is stable,

where Inf $\operatorname{Ric}_{M}$ (resp. Sup $\operatorname{Ric}_{M}$ ) is the infimum (resp. supremum) of the Ricci curvature of $(M, g)$ over $M, \operatorname{Inf} \operatorname{Ric}_{M}:=\operatorname{Inf}\{\rho(u, u) ; u \in T M, g(u, u)=1\}$, and $\operatorname{Sup~Ric}_{M}:=\{\rho(u, u) ; u \in T M, g(u, u)=1\}$.

Proof. By (4.3), the stability of $(M, g)$ implies that

$$
\begin{aligned}
0 & \leqslant \int_{M} g(J V, V) * 1=\int_{M} g(\Delta V, V) * 1-2 \int_{M} g(\rho(V), V) * 1 \\
& \leqslant \int_{M} g(\Delta V, V) * 1-2\left(\operatorname{Inf}_{\operatorname{Ric}_{M}}\right) \int_{M} g(V, V) * 1,
\end{aligned}
$$

which gives the first inequality of (i). Taking $V$ as the gradient of the eigenfunction of $\Delta_{M}$ with the eigenvalue $\lambda_{1}(M)$, we get the second inequaltiy of (i). Statement (ii) is obvious from (4.3). Q.E.D. 
From Lemma 4.1, we obtain

TheOREM 4.2 (M. OвATA). Let $(M, g)$ be a closed Kähler manifold whose Ricci curvature $\mathrm{Ric}_{M}$ is bounded below by a positive constant $\alpha>0$. Then the first nonzero eigenvalue $\lambda_{1}(M)$ of $\Delta_{M}$ on $\mathscr{C}^{\infty}(M)$ satisfies $\lambda_{1}(M) \geqslant 2 \alpha$. When the equality holds, the Lie algebra a of the group of holomorphic transformations of $M$ is nonzero.

Proof. Since every closed Kähler manifold $(M, g)$ is stable (cf. [Sm, Na]), Lemma 4.1(i) gives the inequality $\lambda_{1}(M) \geqslant 2 \alpha$. Assume that the equality $\lambda_{1}(M)=2 \alpha$ holds. We take $V$ as the gradient of the eigenfunction of $\Delta_{M}$ with the eigenvalue $2 \alpha$. Then $\Delta V=2 \alpha V$. By (4.3), we have

$$
\begin{aligned}
2 \alpha \int_{M} g(V, V) * 1 & =\int_{M} g(\Delta V, V) * 1 \\
& =\int_{M} g(J V, V) * 1+2 \int_{M} g(\rho(V), V) * 1 \\
& \geqslant 2 \alpha \int_{M} g(V, V) * 1,
\end{aligned}
$$

since $(M, g)$ is stable and $\operatorname{Ric}_{M} \geqslant \alpha$. Hence we have $\int_{M} g(J V, V) * 1=0$ and $\int_{M} g(\rho(V), V) * 1=\alpha \int_{M} g(V, V) * 1$. The former implies $J V=0$, and then $V$ belongs to a due to a theorem of Lichnérowicz (cf. [L]) since $(M, g)$ is a closed Kähler manifold. Q.E.D.

REMARK 1. In [Ob], the above theorem was stated for a closed Einstein Kähler manifold $(M, g)$. In this case, i.e., $\rho=\alpha g$, the equality $\lambda_{1}(M)=2 \alpha$ holds if and only if $\mathfrak{a} \neq\{0\}$. The author does not know whether or not the equality holds if $a \neq\{0\}$ without the assumption that $(M, g)$ is Einstein.

REMARK 2. A theorem of Lichnérowicz-Obata tells us that for a closed Riemannian manifold $(M, g)$, if $\operatorname{Ric}_{M} \geqslant \alpha=(n-1) \delta>0$, then $\lambda_{1}(M) \geqslant n \delta=$ $n \alpha /(n-1)$. Note that $n /(n-1) \leqslant 2$ and $n /(n-1)=2 \Leftrightarrow n=2$.

5. Some examples. In this section, we give three examples illustrating stability or instability of closed Riemannian manifolds.

5.1. By (4.1) and Corollary 2.2, we know (cf. [Sm]) that if Ricci curvature $\mathrm{Ric}_{M}$ of a closed Riemannian manifold $(M, g)$ is nonpositive, then $\operatorname{Index}\left(\operatorname{id}_{M}\right)=0$ and Index $\left(\mathrm{id}_{M}\right)+\operatorname{Nullity}\left(\mathrm{id}_{M}\right) \leqslant m=\operatorname{dim} M$. Imitating the proof of Proposition 5.6 in [B.G, p. 30], noting only the difference of the constant terms of (4.1) and (4.2), we have

Proposition 5.1. There exists a positive constant $\varepsilon_{m}>0$ depending only on $m$ such that for every closed Riemannian manifold $(M, g)$ of dimension $m$ with $\mathrm{Ric}_{M} \leqslant \varepsilon_{m}$, the index and nullity of the identity map of $M$ satisfy $\operatorname{Index}\left(\operatorname{id}_{M}\right)+\operatorname{Nullity}\left(\mathrm{id}_{M}\right) \leqslant m$.

However one cannot expect to find a positive constant $\varepsilon_{m}>0$ such that for every closed Riemannian manifold $(M, g)$ of dimension $m$ the assumption $\operatorname{Ric}_{M} \leqslant \varepsilon_{m}$ implies the stability of $(M, g)$, i.e., Index $\left(\mathrm{id}_{M}\right)=0$. In fact, we have the following example. 
EXAmple 5.2. Let $\mathbf{T}^{m}=\mathbf{R}^{m} / \mathbf{Z}^{m}$ be the $m$-dimensional torus with the canonical coordinates $\left(x_{1}, \ldots, x_{m}\right)$. Let $f\left(x_{1}\right)$ be a positive valued smooth function on $\mathbf{R} / \mathbf{Z}=$ $S^{1}$. Consider the Riemannian metric $g_{f}$ on $\mathbf{T}^{m}$ defined by

$$
g_{f}:=d x_{1}^{2}+f\left(x_{1}\right)^{2}\left(d x_{2}^{2}+\cdots+d x_{m}^{2}\right) \text {. }
$$

LEMMA 5.3. The vector field $X_{1}=f\left(x_{1}\right) \partial / \partial x_{1}$ on $\mathbf{T}^{m}$ is a conformal vector field, i.e., the Lie derivative $L_{X_{1}} g_{f}$ of $g_{f}$ by $X_{1}$ satisfies $L_{X_{1}} g_{f}=(2 / n) \operatorname{div}\left(X_{1}\right) g_{f}$, and $X_{i}=\partial / \partial x_{i}, i=2, \ldots, m$, are Killing, i.e., $L_{X_{i}} g_{f}=0$.

Proof follows from a straightforward computation.

Since for a vector field $V$ on a closed Riemannian manifold $(M, g)$,

$$
\int_{M} g(J V, V) * 1=\int_{M}\left\{\frac{1}{2}\left|L_{V} g\right|^{2}-\operatorname{div}(V)^{2}\right\} * 1,
$$

where $\left|L_{V} g\right|$ is the norm of $L_{V} g$ induced by $g$ and $\operatorname{div}(V)$ is the divergence of $V$ (cf. [Y.B]), we have

$$
\int_{\mathbf{T}^{m}} g\left(J X_{1}, X_{1}\right) * 1=\left(\frac{2}{m}-1\right) \int_{\mathbf{T}^{m}} \operatorname{div}\left(X_{1}\right)^{2} * 1 .
$$

Since $\operatorname{div}\left(X_{1}\right)=m f^{\prime}\left(x_{1}\right)$ where $f^{\prime}\left(x_{1}\right)$ is the derivative of $f\left(x_{1}\right)$, we have

Proposition 5.4. Let $\mathbf{T}^{m}=\mathbf{R}^{m} / \mathbf{Z}^{m}$ be the $m$-dimensional torus with the canonical coordinates $\left(x_{1}, \ldots, x_{m}\right)$. For a positive valued smooth function $f\left(x_{1}\right)$ on $S^{1}=\mathbf{R} / \mathbf{Z}$, consider the Riemannian metric $g_{f}$ on $\mathbf{T}^{m}$ defined by

$$
g_{f}=d x_{1}^{2}+f\left(x_{1}\right)^{2}\left(d x_{2}^{2}+\cdots+d x_{m}^{2}\right) .
$$

Then, in case $m \geqslant 3$, the Riemannian manifold $\left(\mathbf{T}^{m}, g_{f}\right)$ is stable if and only if the function $f\left(x_{1}\right)$ is constant.

On the other hand the sectional curvature $K$ of the Riemannian manifold $\left(\mathbf{T}^{m}, g_{f}\right)$ is given (cf. [B.O]) as follows:

For each plane $\pi$ in the tangent space $T_{\left(x_{1}, \ldots, x_{m}\right)} \mathbf{T}^{m}$, let $\left\{x \partial / \partial x_{1}+v, y \partial / \partial x_{1}+\right.$ $w$ \} be an orthonormal basis of $\pi$, where $x, y \in \mathbf{R}$, and $v, w \in T_{\left(x_{2}, \ldots, x_{m}\right)} \mathbf{T}^{m-1}$. Then the sectional curvature $K(\pi)$ is

$$
\begin{aligned}
K(\pi)= & -\frac{f^{\prime \prime}\left(x_{1}\right)}{f\left(x_{1}\right)}\left\{x^{2} g_{f}(w, w)-2 x y g_{f}(w, v)+y^{2} g_{f}(v, v)\right\} \\
& -\frac{f^{\prime}\left(x_{1}\right)^{2}}{f\left(x_{1}\right)^{2}}\left\{g_{f}(v, v) g_{f}(w, w)-g_{f}(v, w)^{2}\right\} .
\end{aligned}
$$

Thus the sectional curvature $K$ of $\left(\mathbf{T}^{m}, g_{f}\right)$ satisfies

$$
|K| \leqslant\left|f^{\prime \prime}\right| / f+f^{\prime 2} / f^{2} \text {. }
$$

For example, we can take a smooth function $f_{\varepsilon}\left(x_{1}\right)$ on $S^{1}=\mathbf{R} / \mathbf{Z}$ as $f_{\varepsilon}\left(x_{1}\right):=1+$ $\varepsilon \sin \left(2 \pi x_{1}\right)$, where $\varepsilon$ is a small positive constant. Then due to Proposition 5.4 , the Riemannian manifold $\left(\mathbf{T}^{m}, g_{f_{e}}\right), m \geqslant 3$, is unstable, but its sectional curvature $K_{\varepsilon}$ satisfies

$$
\left|K_{\varepsilon}\right| \leqslant 4 \pi^{2}\left\{\varepsilon /(1-\varepsilon)+\varepsilon^{2} /(1-\varepsilon)^{2}\right\}
$$


which goes to zero as $\varepsilon \rightarrow 0$. Therefore we cannot find a constant $\varepsilon_{m}>0$ such that for every closed Riemannian manifold $(M, g)$ of dimension $m$, the assumption $\operatorname{Ric}_{M} \leqslant \varepsilon_{m}$ implies the stability of $(M, g)$.

5.2. The next example is the odd dimensional unit sphere $S^{2 n+1}, n \geqslant 1$. Let $\phi$; $\left(S^{2 n+1}, g\right) \rightarrow\left(\mathbf{C} P^{n}, h\right)$ be the Hopf fibration. Here $g$ is the standard metric on $S^{2 n+1}$ of constant curvature one and $h$ is the Fubini-Study metric on $\mathbf{C} P^{n}$ of constant holomorphic sectional curvature 4. Let $\xi$ be the Killing vector field on $\left(S^{2 n+1}, g\right)$ such that $g(\xi, \xi)=1$ and $\xi$ is tangent to the fiber $\phi^{-1}(\phi(x))$ at each point $x$ in $S^{2 n+1}$. Let $\eta$ be the 1 -form dual to $\xi$. Then the projection $\phi ;\left(S^{2 n+1}, g\right)$ $\rightarrow\left(\mathbf{C} P^{n}, h\right)$ is a Riemannian submersion with totally geodesic fibers (cf. §6) and $g=\phi^{*} h+\eta \otimes \eta$. Let us consider the canonical variation $g_{t}, 0<t<\infty$, of the metric $g$ defined by

$$
g_{t}:=\phi^{*} h+t^{2} \eta \otimes \eta=g+\left(t^{2}-1\right) \eta \otimes \eta .
$$

Now we investigate the stability of $\left(S^{2 n+1}, g_{t}\right)$ making use of Lemma 4.1.

To estimate the first eigenvalue $\lambda_{1}^{1}\left(g_{t}\right)$ of the Hodge Laplacian, put $m:=2 n+1$. Note that $g_{t}=s\left\{s^{-1} g+s^{-1}\left(s^{m}-1\right) \eta \otimes \eta\right\}$, where $s:=t^{2 / m}$. In his paper [T1, Proposition 2.8], S. Tanno showed that the first eigenvalue $\lambda_{1}^{1}\left(g_{t}\right)$ of the Hodge Laplacian on 1-forms is estimated by

$$
\lambda_{1}^{1}\left(g_{t}\right) \leqslant \operatorname{Min}\left\{s^{-1} \cdot 2(m-1) s^{m+1}, s^{-1}\left(m s-s\left(1-s^{-m}\right)\right)\right\},
$$

that is,

$$
\lambda_{1}^{1}\left(g_{t}\right) \leqslant \operatorname{Min}\left\{4 n t^{2}, 2 n+t^{-2}\right\} .
$$

To study the Ricci curvature of $\left(S^{2 n+1}, g_{t}\right)$, we recall some work of G. R. Jensen [J]. Set

$$
\begin{aligned}
& K:=S U(n+1), \\
& H:=S(U(n) \times U(1))=\left\{\left(\begin{array}{ll}
\varepsilon & 0 \\
0 & A
\end{array}\right) \in S U(n+1) ; \varepsilon \in U(1), A \in U(n)\right\}, \\
& H_{1}:=\left\{\left(\begin{array}{cc}
\varepsilon & 0 \\
0 & \gamma I_{n}
\end{array}\right) ; \varepsilon \in U(1), \gamma=\varepsilon^{-1 / n}\right\}, \\
& H_{2}:=\left\{\left(\begin{array}{ll}
1 & 0 \\
0 & A
\end{array}\right) ; A \in S U(n)\right\},
\end{aligned}
$$

where $I_{n}$ is the unit matrix of order $n$. Then the natural projection gives the Hopf fibration $\phi ; S^{2 n+1}=K / H_{2} \rightarrow \mathbf{C} P^{n}=K / H$. Let $\mathfrak{f}$ (resp. $\mathfrak{h}, \mathfrak{h}_{1}, \mathfrak{h}_{2}$ ) be the Lie algebra of $K$ (resp. $H, H_{1}, H_{2}$ ). Let $F$ be the Killing form of $\mathfrak{f}$ and $\mathfrak{m}$, the orthogonal complement of $\mathfrak{h}$ in $\mathfrak{f}$ with respect to $F$. Then we have the orthogonal decomposition of $\mathfrak{f}: \mathfrak{f}=\mathfrak{h}_{2} \oplus \mathfrak{h}_{1} \oplus \mathfrak{m}$. The metrics $g_{t}$ in (5.1) are $K$-invariant on $K / H_{2}$ and come from the $\operatorname{Ad}\left(H_{2}\right)$-invariant inner product $\langle\cdot, \cdot\rangle_{t}$ on $\mathfrak{h}_{1} \oplus \mathrm{m}$ such that

$$
\left\langle X_{1}+X_{2}, Y_{1}+Y_{2}\right\rangle_{t}=(4(n+1))^{-1}\left\{\frac{2 n}{n+1} t^{2} b\left(X_{1}, Y_{1}\right)+b\left(X_{2}, Y_{2}\right)\right\},
$$


for $X_{1}, Y_{1} \in \mathfrak{h}_{1}, X_{2}, Y_{2} \in \mathfrak{m}$, where the inner product $b$ on $\mathfrak{f}$ is given by $b=-F$. In fact, it is known that the restriction of $b$ to $m$ coincides with $4(n+1) \pi^{*} h$, and $b(X, X)=2(n+1)^{2} / n$ for

$$
X:=\sqrt{-1}\left(\begin{array}{cc}
1 & 0 \\
0 & -n^{-1} I_{n}
\end{array}\right),
$$

and $\xi_{o}$ is the tangent vector at

$$
o:=\left(\begin{array}{c}
1 \\
0 \\
\vdots \\
0
\end{array}\right) \in S^{2 n+1}
$$

of the curve $\theta \mapsto \exp (\theta X) \cdot o$.

We denote by $S_{\tilde{g}}$ the Ricci tensor of the metric $\tilde{g}$ on $K / H_{2}$ corresponding to the inner product $4(n+1)\langle\cdot, \cdot\rangle_{t}$ on $m$. Then $S_{\tilde{g}}$ is a $K$-invariant tensor field on $K / H_{2}$ which is completely determined by the bilinear form on $\mathfrak{h}_{1} \oplus \mathfrak{m}$, denoted by the same letter $S_{\tilde{g}}$. Note that the numbers $k, c, r$, and $\operatorname{dim} m$ in $[\mathbf{J}]$ are given in this case by $k=1 / 2, c=0, r=\operatorname{dim} \mathfrak{h}_{1}=1$, and $\operatorname{dim} m=2 n$. Thus by Proposition 11 in $[\mathbf{J}]$, the bilinear form $S_{\tilde{g}}$ is given by

$$
\begin{aligned}
S_{\tilde{g}}\left(X_{1}+X_{2}, Y_{1}+Y_{2}\right)= & \frac{1}{4}\left(\frac{2 n}{n+1}\right) t^{2} \cdot 4(n+1)\left\langle X_{1}, Y_{1}\right\rangle_{t} \\
& +\left(\frac{1}{2}-\frac{1}{4 n}\left(\frac{2 n}{n+1}\right) t^{2}\right) \cdot 4(n+1)\left\langle X_{2}, Y_{2}\right\rangle_{t},
\end{aligned}
$$

$X_{1}, Y_{1} \in \mathfrak{h}_{1}, X_{2}, Y_{2} \in \mathrm{m}$. Since

$$
\begin{gathered}
\operatorname{Inf}_{\operatorname{Ric}_{\tilde{g}}}=\operatorname{Min}\left\{\frac{1}{2}-\frac{t^{2}}{2(n+1)}, \frac{n}{2(n+1)} t^{2}\right\}, \\
\operatorname{Sup} \operatorname{Ric}_{\tilde{g}}=\operatorname{Max}\left\{\frac{1}{2}-\frac{t^{2}}{2(n+1)}, \frac{n}{2(n+1)} t^{2}\right\},
\end{gathered}
$$

it follows that

$$
\begin{aligned}
& \operatorname{Inf} \operatorname{Ric}_{g_{t}}=\operatorname{Min}\left\{2(n+1)-2 t^{2}, 2 n t^{2}\right\}, \\
& \operatorname{Sup~Ric}_{g_{t}}=\operatorname{Max}\left\{2(n+1)-2 t^{2}, 2 n t^{2}\right\} .
\end{aligned}
$$

To interpret (5.2) and (5.3), see Figure 5.1 in which $T=t^{2}$. Therefore we have

PROPOSITION 5.5. Let $g_{t}$ be the canonical variation (5.1) of the standard metric $g$ of constant curvature one on $S^{2 n+1}$ with $g_{t}=g+\left(t^{2}-1\right) \eta \otimes \eta$. Then for every $t^{2}$ in the open interval $(\alpha, \beta)$, the Riemannian manifold $\left(S^{2 n+1}, g_{t}\right)$ is unstable. Here $\alpha:=$ $\left(n+\sqrt{n^{2}+4 n}\right) / 4 n\left(\right.$ resp. $\left.\beta:=\left(n+2+\sqrt{n^{2}+4 n}\right) / 4\right)$ is a root of the equation $4 n T=2 n+T^{-1}\left(\operatorname{resp} .4(n+1)-4 T=2 n+T^{-1}\right)$. 


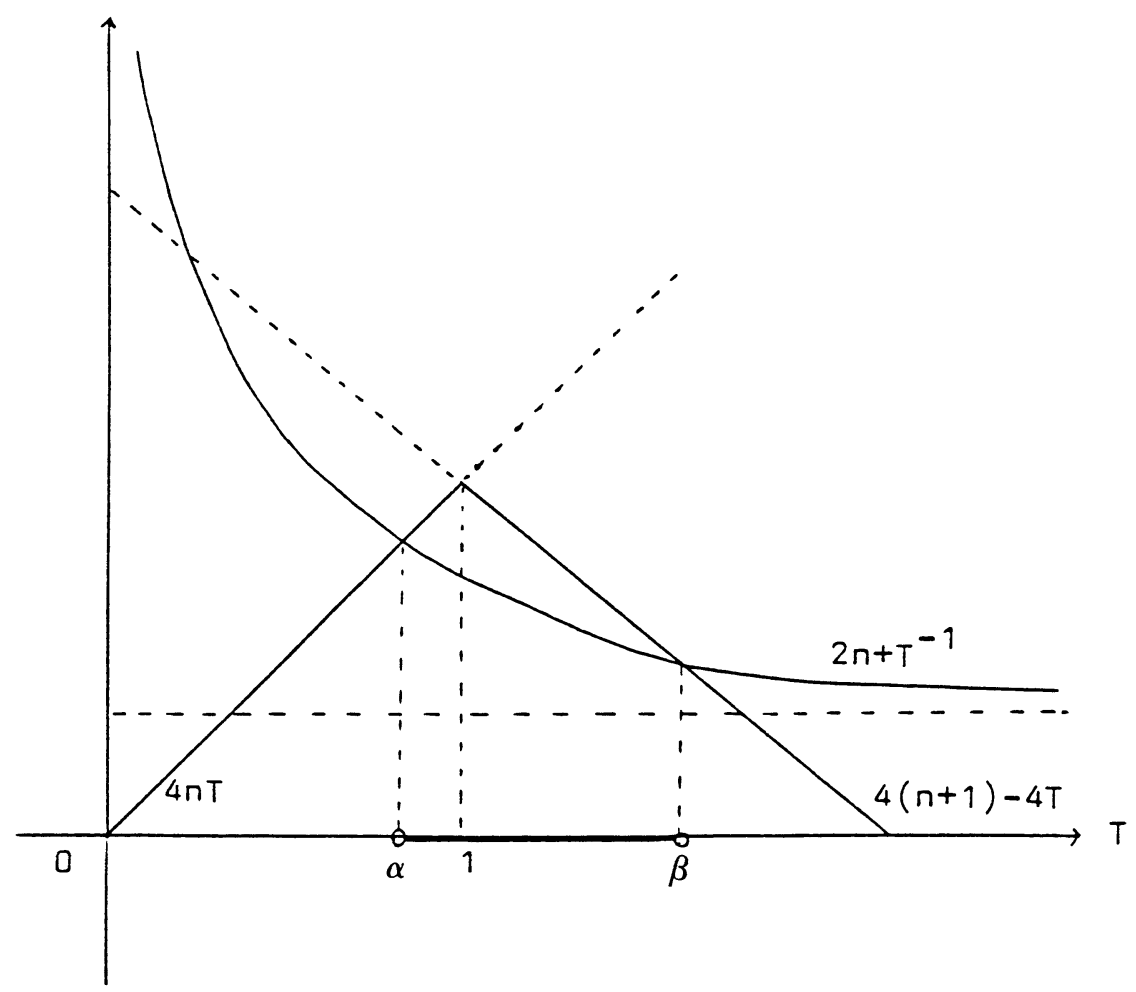

Figure 5.1. The graphs of the functions $4 n T, 2 n+T^{-1}$, and $4(n+1)-4 T$

5.3. The third example is a spherical space form. Here we state the following:

Proposition 5.6. Every spherical space form $\left(S^{n} / G, g\right)$, where $G \neq\{\mathrm{id}\}$ is a finite group acting fixed point freely on $S^{n}$, is stable. Here the metric $g$ is the Riemannian metric on the quotient space $S^{n} / G$ induced by the standard metric can of constant curvature one on $S^{n}$.

In fact, this follows immediately from Proposition 2.1 in [Sm]. Since $\left(S^{n} / G, g\right)$ is Einstein, i.e., the Ricci tensor $\rho$ of $g$ satisfies $\rho=(n-1) g$, the manifold $\left(S^{n} / G, g\right)$ is stable if and only if the first nonzero eigenvalue $\lambda_{1}\left(S^{n} / G, g\right)$ of the LaplaceBeltrami operator $\Delta_{M}$ on $\mathscr{C}^{\infty}\left(S^{n} / G\right)$ is bigger than or equal to $2(n-1)$. The eigenvalues of $\Delta_{M}$ of $\left(S^{n}\right.$, can $)$ are given by $k(k+n-1), k=0,1,2, \ldots$, and $k(k+n-1)>2(n-1)$ if $k \geqslant 2$. Moreover the eigenfunctions of the first nonzero eigenvalue $n$ with $k=1$ of $\left(S^{n}\right.$, can) are given by $F \cdot \mathrm{id}_{S^{n}}$, where $F$ is a linear map of $\mathbf{R}^{n+1}$ into $\mathbf{R}$ and id $S^{n}$ is the natural inclusion of $S^{n}$ into $\mathbf{R}^{n+1}$. Therefore we only have to show that every linear $G$-invariant function $F$ on $\mathbf{R}^{n+1}$ must be zero. But this follows immediately from the assumption that $G$ acts fixed point freely on $S^{n}$. Certainly, $F(x)=\langle x, y\rangle, x \in \mathbf{R}^{n+1}$, for some $y$ in $\mathbf{R}^{n+1}$. The $G$-invariance of $F$ implies that $\gamma \cdot y=y$ for all $\gamma \in G$. Unless $F$ vanishes, the point $y /|y| \in S^{n}$ must be a fixed point of $G$. 
Since every compact Riemannian manifold of positive constant curvature is as in Proposition 5.6 (cf. [W, Lemma 5.11, p. 154]) and every compact Riemannian manifold of constant zero or negative curvature is stable (cf. [Sm]), we have

COROLlARY 5.7. Every compact Riemannian manifold of constant curvature is stable except for the standard unit sphere $\left(S^{n}\right.$, can $)$.

REMARK. A similar stability theorem for Yang-Mills fields was stated in [B.L, p. 223].

\section{CHAPTER III. RIEMANNIAN SUBMERSIONS WITH TOTALLY GEODESIC FIBERS}

\section{The vertical Jacobi operator.}

6.1. Following [O.N] or [B.B], let us recall the definition of a Riemannian submersion. It is known (cf. [E.L, p. 127]) that the projection of a Riemannian submersion is harmonic if and only if each fiber of the submersion is a minimal submanifold. In particular, the projection of the Riemannian submersion with totally geodesic fibers is harmonic. The Riemannian submersions are the next simple examples after Riemannian products, but would be rich objects to study. In this chapter, we study Jacobi operators of projections of Riemannian submersions with totally geodesic fibers by analogy with the theory of Laplace-Beltrami operators (cf. [B.B]).

Definition 6.1. Let $(M, g)$ and $(N, h)$ be two closed Riemannian manifolds of dimension $m$ and $n$ respectively. A map $\phi ;(M, g) \rightarrow(N, h)$ is a Riemannian submersion (cf. [O.N, B.B]) if for each point $p$ in $M$, the tangent space $T_{p} M$ of $M$ at $p$ has an orthogonal decomposition $T_{p} M=H_{p} \oplus V_{p}$ with respect to $g_{p}$ such that

(i) the subspace $V_{p}$ is the kernel of the differential $\phi_{*_{p}}$ of $\phi$ at $p$, which is called the vertical space, and

(ii) the restriction of $\phi_{*_{p}}$ to the subspace $H_{p}$, called the horizontal space, is an isometry of $\left(H_{p}, g_{p}\right)$ onto $\left(T_{\phi(p)} N, h_{\phi(p)}\right)$. A vector field $X$ on $U \subset M$ is called basic if it is the horizontal lift of a vector field $X^{\prime}$ on $\phi(U) \subset N$. In this chapter, we further assume that each fiber $F_{p}:=\phi^{-1}(\phi(p))$ through $p$ admitting the Riemannian metric induced from $g$ is totally geodesic in $(M, g)$.

6.2. To define the vertical Jacobi operator, we take an orthonormal local frame field $\left\{e_{i}\right\}_{i=1}^{m}$ on $M$ such that

(i) for $1 \leqslant i \leqslant n$, each $e_{i}$ is basic, the horizontal lift of $e_{i}^{\prime}$, with $\left\{e_{i}^{\prime}\right\}_{i=1}^{m}$ an orthonormal local frame field on $N$, and

(ii) for $n+1 \leqslant i \leqslant m$, each $e_{i}$ is vertical.

Then it is known (cf. [O.N or B.B]) that $\nabla_{e_{i}} e_{i}, 1 \leqslant i \leqslant n$, is basic, the horizontal lift of ${ }^{N} \nabla_{e_{i}^{\prime}} e_{i}^{\prime}$, while $\nabla_{e_{i}} e_{i}, n+1 \leqslant i \leqslant m$, is vertical since all the fibers are totally geodesic. In the following we retain the notation of $\$ 1$.

DEFINITION 6.2. Let $\phi ;(M, g) \rightarrow(N, h)$ be a Riemannian submersion with totally geodesic fibers and $J_{\phi}$, the Jacobi operator acting on $\Gamma\left(\phi^{-1} T N\right)$. We define the vertical Jacobi operator acting on $\Gamma\left(\phi^{-1} T N\right)$ by

$$
J_{\phi}^{v}:=-\sum_{i=n+1}^{m}\left(\tilde{\nabla}_{e_{i}} \tilde{\nabla}_{e_{i}}-\tilde{\nabla}_{\nabla_{e_{i}} e_{i}}\right) \text {, }
$$


and the horizontal Jacobi operator acting on $\Gamma\left(\phi^{-1} T N\right)$ by $J_{\phi}^{H}:=J_{\phi}-J_{\phi}^{v}$. Then it is easy to see that the definitions of $J_{\phi}^{v}$ and $J_{\phi}^{H}$ do not depend on the above choice of the orthonormal local frame field $\left\{e_{i}\right\}_{i=1}^{m}$ on $M$ (cf. Remark below). These definitions give analogues of the vertical and horizontal Laplacians $\Delta_{v}$ and $\Delta_{H}$ acting on $\mathscr{C}^{\infty}(M)$ defined in [B.B] by $\Delta_{v}:=\sum_{i=n+1}^{m}\left(\nabla_{e_{i}} \nabla_{e_{i}}-\nabla_{\nabla_{e_{i}} e_{i}}\right)$ and $\Delta_{H}:=\Delta_{M}-\Delta_{v}$, where $\Delta_{M}:=\sum_{i=1}^{m}\left(\nabla_{e_{i}} \nabla_{e_{i}}-\nabla_{\nabla_{e_{i}} e_{i}}\right)$ is the Laplacian-Beltrami operator of $(M, g)$. Note that $\Delta_{v}, \Delta_{H}$, and $\Delta_{M}$ commute (cf. [B.B, Theorem 1.5]).

Each section $W$ in $\Gamma\left(\phi^{-1} T N\right)$ can be expressed locally as

$$
W=\sum_{i=1}^{n} \tilde{f_{i}} \widetilde{e_{i}^{\prime}}
$$

where the $f_{i}, 1 \leqslant i \leqslant n$, are locally defined smooth functions on $M$ and the $\widetilde{e_{i}^{\prime}}$, $1 \leqslant i \leqslant n$, are local sections of $\phi^{-1} T N$ defined by $\widetilde{e_{i x}^{\prime}}:=e_{i \phi(x)}^{\prime}, x \in M$. Then by definition of $\tilde{\nabla}$ and $\phi_{*} e_{i}=0, n+1 \leqslant i \leqslant m$, we have

$$
\begin{gathered}
\tilde{\nabla}_{e_{i}} W=\sum_{j=1}^{n}\left\{\left(e_{i} f_{j}\right) \widetilde{e_{j}^{\prime}}+f_{j} \tilde{\nabla}_{e_{i}} \widetilde{e_{j}^{\prime}}\right\}, \quad 1 \leqslant i \leqslant m, \\
\tilde{\nabla}_{e_{i}} W=\sum_{j=1}^{n}\left(e_{i}^{\prime} f_{j}\right) \widetilde{e_{j}^{\prime}}, \quad n+1 \leqslant i \leqslant m .
\end{gathered}
$$

In particular,

$$
J_{\phi}^{v} W=-\sum_{j=1}^{n}\left(\Delta_{v} f_{j}\right) \widetilde{e_{j}^{\prime}} .
$$

REMARK. The intrinsic meaning of the vertical Jacobi operator is described as follows. For each fiber $F_{p}=\phi^{-1}(\phi(p))$ through $p \in M$, the composition $\phi \circ i_{p}$; $F_{p} \rightarrow N$ of the inclusion $i_{p}$ of $F_{p}$ into $M$ and the projection $\phi$ is constant, so harmonic. The associate Jacobi operator $J_{\phi \circ i_{p}}$ acting on $\Gamma\left(\left(\phi \circ i_{p}\right)^{-1} T N\right)$ is well defined. Then $\Gamma\left(\left(\phi \circ i_{p}\right)^{-1} T N\right)$ consists of all the restrictions, $\left.W\right|_{F_{p}}$, to $F_{p}$ of elements $W$ in $\Gamma\left(\phi^{-1} T N\right)$ and

$$
\left(J_{\phi}^{v} W\right)(p)=J_{\phi \circ i_{p}}\left(\left.W\right|_{F_{p}}\right)(p), \quad W \in \Gamma\left(\phi^{-1} T N\right) .
$$

6.3. We now describe some fundamental properties of $J_{\phi}^{v}$ and $J_{\phi}^{H}$. Note that, by the definitions of $\tilde{\nabla}$ and $\widetilde{W^{\prime}}$,

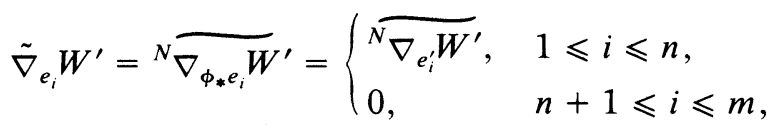

for $W^{\prime} \in \Gamma(T N)$. Then we have

$$
J_{\phi}^{v}\left(\widetilde{W^{\prime}}\right)=0, \text { and } J_{\phi}^{H}\left(\widetilde{W^{\prime}}\right)=\widetilde{J_{\text {id }_{N}}\left(W^{\prime}\right)},
$$

for $W^{\prime} \in \Gamma(T N)$, by (6.4) and definition of $J_{\phi}^{v}$ and $J_{\phi}^{H}$. Therefore we obtain

Proposition 6.3. Let $\phi ;(M, g) \rightarrow(N, h)$ be a Riemannian submersion with totally geodesic fibers. Then

$$
\operatorname{Index}(\phi) \geqslant \operatorname{Index}\left(\mathrm{id}_{N}\right), \quad \operatorname{Nullity}(\phi) \geqslant \operatorname{Nullity}\left(\mathrm{id}_{N}\right),
$$

and $\lambda_{1}\left(J_{\phi}\right) \leqslant \lambda_{1}\left(J_{\mathrm{id}_{N}}\right)$. In particular, if the base manifold $(N, h)$ is unstable, then the submersion $\phi$ is unstable. 
Proof follows immediately from definitions and (6.5).

REMARK. The referee pointed out that Proposition 6.3 was obtained independently by M. J. Ferreira in his Ph.D. Thesis. Moreover, throughout $\S \S 6.1-6.3$ the assumption that the fibers of the Riemannian submersions must be totally geodesic can be replaced by the assumption that the Riemannian submersions are harmonic.

Proposition 6.4. (i) Let $F=F_{p}$ be the fiber through $p \in M$ of a Riemannian submersion $\phi ;(M, g) \rightarrow(N, h)$ with totally geodesic fibers. For each $W \in \Gamma\left(\phi^{-1} T N\right)$, we have

$$
\int_{F} h\left(J_{\phi}^{v} W, W\right) d v_{F}=\sum_{i=n+1}^{m} \int_{F} h\left(\tilde{\nabla}_{e_{i}} W, \tilde{\nabla}_{e_{i}} W\right) d v_{F},
$$

where $d v_{F}$ is the volume element on $F$ with respect to the metric $g_{F}$ induced by the metric $g$ on $M$.

(ii) Moreover, for each $W \in \Gamma\left(\phi^{-1} T N\right), J_{\phi}^{v} W=0$ if and only if $W=\widetilde{W^{\prime}}$ for some $W^{\prime} \in \Gamma(T N)$.

(iii) Each eigenvalue of $J_{\phi}^{v}$ is nonnegative.

Proof. (i) For each $W \in \Gamma\left(\phi^{-1} T N\right)$, we have

$$
\begin{aligned}
h\left(J_{\phi}^{v} W, W\right)= & -\sum_{i=n+1}^{m} e_{i} \cdot h\left(\tilde{\nabla}_{e_{i}} W, W\right)+\sum_{i=n+1}^{m} h\left(\tilde{\nabla}_{e_{i}} W, \tilde{\nabla}_{e_{i}} W\right) \\
& +\sum_{i=n+1}^{m} h\left(\tilde{\nabla}_{\nabla_{e_{i}} e_{i}} W, W\right) .
\end{aligned}
$$

Here there exists an element $X$ in $\Gamma(T F)$ such that $g_{F}(X, Y)=h\left(\tilde{\nabla}_{Y} W, W\right)$ for each $Y \in \Gamma(T F)$. Then since $\nabla_{e_{i}} e_{i}, n+1 \leqslant i \leqslant m$, are vertical

$$
\begin{aligned}
& \sum_{i=n+1}^{m}\left\{e_{i} \cdot h\left(\tilde{\nabla}_{e_{i}} W, W\right)-h\left(\tilde{\nabla}_{\nabla_{e_{i}} e_{i}} W, W\right)\right\} \\
& =\sum_{i=n+1}^{m}\left\{e_{i} \cdot g_{F}\left(X, e_{i}\right)-g_{F}\left(\nabla_{e_{i}} e_{i}, X\right)\right\}
\end{aligned}
$$

is the gradient of $X$ on $\left(F, g_{F}\right)$. Therefore we have (i).

(ii) By (6.5), we only have to prove that if $J_{\phi}^{v} W=0$, then $W=\widetilde{W^{\prime}}$ for some $W^{\prime} \in \Gamma(T N)$. Assume that $J_{\phi}^{v} W=0$. Then by (i) we have $\tilde{\nabla}_{e_{i}} W=0, n+1 \leqslant i \leqslant m$. We choose a local coordinate system $\left(x_{U}^{1}, \ldots, x_{U}^{n}\right)$ on a neighborhood $U$ in $N$. Then $W$ can be expressed locally as

$$
W_{x}=\sum_{j=1}^{n} f_{U, j}(x)\left(\frac{\partial}{\partial x_{U}^{j}}\right)_{\phi(x)}, \quad x \in \phi^{-1}(U),
$$

where $f_{U, j} \in \mathscr{C}^{\infty}\left(\phi^{-1}(U)\right)$. Since $W \in \Gamma\left(\phi^{-1} T N\right)$, it satisfies

$$
f_{U, i}=\sum_{j=1}^{n} f_{V, j} \frac{\partial x_{U}^{i}}{\partial x_{V}^{j}}
$$


on $\phi^{-1}(U) \cap \phi^{-1}(V)$ for another coordinate system $\left(x_{V}^{1}, \ldots, x_{V}^{n}\right)$ on $V$. By $\left(6.2^{\prime}\right)$ $0=\tilde{\nabla}_{e_{i}} W=\sum_{j=1}^{n}\left(e_{i} f_{U, j}\right)\left(\overline{\partial / \partial x_{U}^{j}}\right)$. Therefore $e_{i} f_{U, j}=0, n+1 \leqslant i \leqslant m$, that is, the $f_{U, j}$ are constant along each fiber, which implies that $f_{U, j}=f_{U, j}^{\prime}{ }^{\circ} \phi$ for some $f_{U, j}^{\prime,} \in \mathscr{C}^{\infty}(U)$. By (6.6), $f_{U, j}^{\prime}$ satisfies

$$
f_{U, i}^{\prime}=\sum_{j=1}^{n} f_{V, j}^{\prime}\left(\partial x_{U}^{i} / \partial x_{V}^{j}\right) \quad \text { on } U \cap V
$$

Therefore $\left\{\sum_{j=1}^{n} f_{U, j}^{\prime} \partial / \partial x_{U}^{j}\right\}$ defines a section $W^{\prime}$ in $\Gamma(T N)$ such that $W=\widetilde{W^{\prime}}$.

Finally, (iii) follows immediately from (i). Q.E.D.

6.4. This section is devoted to the following result.

THEOREM 6.5. Let $\phi ;(M, g) \rightarrow(N, h)$ be a Riemannian submersion with totally geodesic fibers. Then the operators $J_{\phi}^{v}, J_{\phi}^{H}$ and $J_{\phi}$ commute.

PRoof. We only have to prove that $J_{\phi}^{v} J_{\phi}^{H}=J_{\phi}^{H} J_{\phi}^{v}$. For each $W \in \Gamma\left(\phi^{-1} T N\right)$, we have

$$
\begin{aligned}
J_{\phi}^{H} J_{\phi}^{v} W= & -\sum_{j, k=1}^{n}\left\{\left(\tilde{\nabla}_{e_{k}} \tilde{\nabla}_{e_{k}}-\tilde{\nabla}_{\nabla_{e_{k}} e_{k}}\right)\left(\left(\Delta_{v} f_{j}\right) \widetilde{e_{j}^{\prime}}\right)+\left(\Delta_{v} f_{j}\right){ }^{N} R\left(e_{k}^{\prime}, e_{j}^{\prime}\right) e_{k}^{\prime}\right\} \\
= & -\sum_{j, k=1}^{n}\left\{e_{k}^{2}\left(\Delta_{v} f_{j}\right) \widetilde{e_{j}^{\prime}}+2 e_{k}\left(\Delta_{v} f_{j}\right) \tilde{\nabla}_{e_{k}} e_{j}^{\prime}+\left(\Delta_{v} f_{j}\right) \tilde{\nabla}_{e_{k}} \tilde{\nabla}_{e_{k}} \widetilde{e_{j}^{\prime}}\right. \\
& \left.\quad-\left(\nabla_{e_{k}} e_{k}\right)\left(\Delta_{v} f_{j}\right) \widetilde{e_{j}^{\prime}}-\left(\Delta_{v} f_{u}\right) \tilde{\nabla}_{\nabla_{e_{k}} e_{k}} \widetilde{e_{j}^{\prime}}\right\} \\
& -\sum_{j, k=1}^{n}\left(\Delta_{v} f_{j}\right){ }^{N} R\left(e_{k}^{\prime}, e_{j}^{\prime}\right) e_{k}^{\prime},
\end{aligned}
$$

by definition of $J_{\phi}^{H}$ and (6.3). Since the $e_{k}$ and $\nabla_{e_{k}} e_{k}, 1 \leqslant k \leqslant n$, are basic, and $\Delta_{v}$ commutes with basic vector fields (cf. [B.B, Lemma 1.6]), the first term of the right-hand side of (6.7) becomes

$$
\begin{gathered}
-\sum_{j, k=1}^{n}\left\{\Delta_{v}\left(e_{k}^{2} f_{j}\right) \widetilde{e_{j}^{\prime}}+2 \Delta_{v}\left(e_{k} f_{j}\right) \tilde{\nabla}_{e_{k}} \widetilde{e_{j}^{\prime}}+\left(\Delta_{v} f_{j}\right) \tilde{\nabla}_{e_{k}} \tilde{\nabla}_{e_{k}} \widetilde{e_{j}^{\prime}}\right. \\
\left.-\Delta_{v}\left(\nabla_{e_{k}} e_{k} f_{j}\right) \widetilde{e_{j}^{\prime}}-\left(\Delta_{v} f_{j}\right) \tilde{\nabla}_{\nabla_{e_{k}} e_{k}} \widetilde{e_{j}^{\prime}}\right\} \\
=-\sum_{j, k=1}^{n} J_{\phi}^{v}\left\{\left(e_{k}^{2} f_{j}\right) \widetilde{e_{j}^{\prime}}+2\left(e_{k} f_{j}\right) \tilde{\nabla}_{e_{k}} \widetilde{e_{j}^{\prime}}+f_{j} \tilde{\nabla}_{e_{k}} \tilde{\nabla}_{e_{k}} \widetilde{e_{j}^{\prime}}\right. \\
\left.-\left(\nabla_{e_{k}} e_{k} f_{j}\right) \widetilde{e_{j}^{\prime}}-f_{j} \tilde{\nabla}_{\nabla_{e_{k}} e_{k}} \widetilde{e_{j}^{\prime}}\right\},
\end{gathered}
$$

by (6.3) and (6.4). Therefore we obtain

$$
J_{\phi}^{H} J_{\phi}^{v} W=-\sum_{k=1}^{n} J_{\phi}^{v}\left\{\left(\tilde{\nabla}_{e_{k}} \tilde{\nabla}_{e_{k}}-\tilde{\nabla}_{\nabla_{e_{k}} e_{k}}\right) W-{ }^{N} R\left(e_{k}^{\prime}, W\right) e_{k}^{\prime}\right\}=J_{\phi}^{v} J_{\phi}^{H} W
$$

Q.E.D. 
We have immediately

COROLlaRY 6.6. The Hilbert space of all $L^{2}$ sections of $\phi^{-1} T N$ with respect to the inner product $(V, W):=\int_{M} h(V, W) * 1$, for sections $V, W$, has a complete orthonormal basis consisting of the simultaneous eigensections of $J_{\phi}^{v}, J_{\phi}^{H}$, and $J_{\phi}$.

\section{The canonical variation of a Riemannian submersion.}

7.1. We continue the discussion in $\S 6$. Let $\phi ;(M, g) \rightarrow(N, h)$ be a Riemannian submersion with totally geodesic fibers.

Definition 7.1 (cf. [B.B, p. 191]). For each positive real number $t$, let $g_{t}$ be the unique Riemannian metric on $M$ such that

(i) $g_{t}(u, v)=g(u, v)$ for $u, v \in H_{p}, p \in M$,

(ii) the subspaces $H_{p}$ and $V_{p}$ are orthogonal to each other with respect to $g_{t}$ at each point $p$ in $M$, and

(iii) $g_{t}(u, v)=t^{2} g(u, v)$ for $u, v \in V_{p}, p \in M$.

Then $\phi ;\left(M, g_{t}\right) \rightarrow(N, h)$ is a Riemannian submersion with totally geodesic fibers (cf. [B.B, Proposition 5.2]), which is called the canonical variation.

For each $t>0,\left\{e_{1}, \ldots, e_{n}, t^{-1} e_{n+1}, \ldots, t^{-1} e_{m}\right\}$ is an orthonormal local frame field on $\left(M, g_{t}\right)$ with $e_{i}$ the horizontal lift of $e_{i}^{\prime}$ with respect to $g_{t}$ for $1 \leqslant i \leqslant n$, and with $t^{-1} e_{i}$ vertical for $n+1 \leqslant i \leqslant n$. Then the vertical (resp. horizontal) Jacobi operator ${ }^{t} J_{\phi}^{v}$ (resp. $\left.{ }^{t} J_{\phi}^{H}\right)$ of the canonical variation $\phi ;\left(M, g_{t}\right) \rightarrow(N, h)$ satisfies

$$
{ }^{t} J_{\phi}^{v}=t^{-2} J_{\phi}^{v}, \quad \text { and } \quad{ }^{t} J_{\phi}^{H}=J_{\phi}^{H} .
$$

Therefore we have

PROPOSITION 7.2. The following formula holds:

$$
{ }^{t} J_{\phi}=t^{-2} J_{\phi}^{v}+J_{\phi}^{H}=t^{-2} J_{\phi}+\left(1-t^{-2}\right) J_{\phi}^{H} .
$$

REMARK. This is the analogue of Proposition 5.3 in [B.B].

7.2. Due to Corollary 6.6 and Proposition 7.2, each eigenvalue of ${ }^{t} J_{\phi}$ can be written as

$$
\lambda+t^{-2} \mu
$$

where $\lambda$ is an eigenvalue of $J_{\phi}^{H}$ and $\mu \geqslant 0$ is an eigenvalue of $J_{\phi}^{v}$. Then the following two cases occur:

(i) $\mu>0$, or

(ii) $\mu=0$.

In case (i), $\lambda+t^{-2} \mu$ goes to infinity when $t \rightarrow 0$. In case (ii), $\lambda+t^{-2} \mu=\lambda$ which does not depend on $t$. Since the number of the eigenvalues of $J_{\phi}$ smaller than a given number is finite, there exists a small positive number $\varepsilon$ such that for each $0<t<\varepsilon$, the first eigenvalue $\lambda_{1}\left({ }^{t} J_{\phi}\right)$ coincides with the smallest eigenvalue of ${ }^{t} J_{\phi}$ when the case (ii) occurs. Then we have

$$
\begin{aligned}
\lambda_{1}\left({ }^{t} J_{\phi}\right) & =\operatorname{Min}\left\{\lambda ; J_{\phi} W=\lambda W \text { and } J_{\phi}^{v} W=0 \text { for some } 0 \neq W \in \Gamma\left(\phi^{-1} T N\right)\right\} \\
& =\lambda_{1}\left(J_{\mathrm{id}_{N}}\right),
\end{aligned}
$$


because of Propositions 6.4(ii) and 6.3. Therefore we obtain

THEOREM 7.3. Let $\phi ;(M, g) \rightarrow(N, h)$ be a Riemannian submersion with totally geodesic fibers, and let $g_{t}, 0<t<\infty$, be the canonical variation ( $c f$. Definition 7.1) of $g$ with $g_{1}=g$. Then there exists a number $\varepsilon>0$ such that for each $0<t<\varepsilon$,

$$
\lambda_{1}\left({ }^{t} J_{\phi}\right)=\lambda_{1}\left(J_{\mathrm{id}_{N}}\right) \text {. }
$$

In particular, if $(N, h)$ is stable, then the submersion $\phi ;(M, g) \rightarrow(N, h)$ is stable for every $0<t<\varepsilon$.

7.3. Typical examples of a Riemannian submersion with totally geodesic fibers are the homogeneous Riemannian submersions (cf. [B.B, §2]). Let $G$ be a compact connected Lie group, and $K, H$ closed subgroups of $G$. Let $\mathfrak{g}$ (resp. $\mathfrak{f}, \mathfrak{h}$ ) be the Lie algebra of $G$ (resp. $K, H$ ). We choose subspaces $\mathfrak{h}_{1}$ (resp. $\mathfrak{p}$ ) of $\mathfrak{f}$ (resp. $\mathfrak{g}$ ) such that $\mathfrak{f}=\mathfrak{h} \oplus \mathfrak{h}_{1}$, with $\operatorname{Ad}(H) \mathfrak{h}_{1}=\mathfrak{h}_{1}$, and $\mathfrak{g}=\mathfrak{l} \oplus \mathfrak{p}$, with $\operatorname{Ad}(K) \mathfrak{p}=\mathfrak{p}$. Put $\mathfrak{m}:=\mathfrak{h}_{1} \oplus \mathfrak{p}$. Then $\mathfrak{g}=\mathfrak{h} \oplus \mathfrak{m}$, with $\operatorname{Ad}(H) \mathfrak{m}=\mathfrak{m}$. Let $(\cdot, \cdot)_{\mathfrak{h}_{1}}$ (resp. $\left.(\cdot, \cdot)_{\mathfrak{p}}\right)$ be an $\operatorname{Ad}(H)$-invariant (resp. Ad( $K)$-invariant) inner product on $\mathfrak{h}_{1}$ (resp. $\mathfrak{p}$ ). We define an $\operatorname{Ad}(H)$-invariant inner product $(\cdot, \cdot)_{\mathfrak{m}}$ on $\mathfrak{m}$ by

$$
\left(X_{1}+X_{2}, Y_{1}+Y_{2}\right)_{\mathfrak{m}}:=\left(X_{1}, Y_{1}\right)_{\mathfrak{h}_{1}}+\left(X_{2}, Y_{2}\right)_{\mathfrak{p}}, \quad X_{1}, Y_{1} \in \mathfrak{h}_{1}, X_{2}, Y_{2} \in \mathfrak{p} .
$$

Then the inner product $(\cdot, \cdot)_{\mathfrak{h}_{1}}\left(\operatorname{resp} .(\cdot, \cdot)_{\mathfrak{p}},(\cdot, \cdot)_{\mathfrak{m}}\right)$ gives a $K$-invariant (resp. $G$-invariant) Riemannian metric $k$ (resp. $h, g$ ) on $K / H$ (resp. $G / K, G / H$ ). It is known (cf. [B.B]) that the projection $\phi ; G / H \ni x H \mapsto x K \in G / K$ gives a Riemannian submersion of $(G / H, g)$ onto $(G / K, h)$ with totally geodesic fibers $(K / H, k)$.

In particular, these give the Hopf fibrations:

(i) $\phi_{1} ; S^{4 n+3}=S p(n+1) / S p(n) \rightarrow \mathbf{H} P^{n}=S P(n+1) / S p(1) \times S p(n)$,

(ii) $\phi_{2} ; S^{2 n+1}=S U(n+1) / S U(n) \rightarrow \mathbf{C} P^{n}=S U(n+1) / S(U(1) \times U(n))$.

Note that $\mathrm{Sp}(n+1)$-invariant (resp. $\mathrm{SU}(n+1)$-invariant) metrics $h$ on $\mathbf{H} P^{n}$ (resp. $\left.\mathrm{C} P^{n}\right)$ are unique up to a constant factor.

Since $\left(\mathbf{H} P^{n}, h\right)\left(\right.$ resp. $\left.\left(\mathbf{C} P^{n}, h\right)\right)$ is unstable (resp. stable) (cf. [Sm, Na]), we have

Proposition 7.4. (i) For each $S p(n+1)$-invariant metric $g$ on $S^{4 n+3}=S p(n+$ 1)/Sp $(n)$, the Riemannian submersion $\phi_{1} ;\left(S^{4 n+3}, g\right) \rightarrow\left(\mathbf{H} P^{n}, h\right)$ is unstable.

(ii) For each $S U(n+1)$-invariant metric $g$ on $S^{2 n+1}=S U(n+1) / S U(n)$, there exists a number $\varepsilon>0$ such that for each $0<t<\varepsilon$, the canonical variation $\phi_{2}$; $\left(S^{2 n+1}, g_{t}\right) \rightarrow\left(\mathbf{C} P^{n}, h\right)$ is stable.

The proof follows from Proposition 6.3 and Theorem 7.3.

REMARK. Proposition 7.4 asserts that each odd dimensional unit sphere $S^{2 n+1}$, $n \geqslant 1$, with the canonical variation $g_{t}, 0<t<\varepsilon$, admits a nonconstant stable harmonic map. By way of contrast, Y. L. Xin [X] showed that each nonconstant harmonic map from the standard unit sphere $\left(S^{m}\right.$, can $), m \geqslant 3$, of constant curvature into an arbitrary Riemannian manifold is unstable.

7.4. Next, let us study the case in which $t$ goes to infinity. We retain the notation of $\$ 7.1$. Let us recall that the holonomy group $G$ of a fiber $F$ of the submersion $\phi$; $(M, g) \rightarrow(N, h)$ with totally geodesic fibers is the group of all isometries of the fiber $F$ induced by the horizontal transports along the horizontal lifts of loops in $N$ based 
at the projection of $F$. It is known [O.N, Theorem 5] that $G=\{$ id $\}$ if and only if the submersion $\phi ;(M, g) \rightarrow(N, h)$ is trivial, that is, there exist an isometry $\iota$ of $(M, g)$ and a submanifold $F$ of $M$ such that $M$ is the Riemannian product $F \times N$ and $\phi=$ pro $\iota$, where pr is the projection of $F \times N$ onto $N$.

THEOREM 7.5. Let $\phi ;(M, g) \rightarrow(N, h)$ be a Riemannian submersion with totally geodesic fibers. Assume that the holonomy group $G$ of a fiber $F$ of the submersion $\phi$; $(M, g) \rightarrow(N, h)$ does not act transitively on the fiber, and $\operatorname{Index}\left(\operatorname{id}_{N}\right)>0$. Then the index of the canonical variation $\phi ;\left(M, g_{t}\right) \rightarrow(N, h)$ goes to infinity when $t \rightarrow \infty$.

Proof. Let $\mathscr{C}_{G}^{\infty}(F)$ be the space of all functions $f$ in $\mathscr{C}^{\infty}(F)$ invariant under the actions of $G$. Since each $G$-orbit has an open $G$-invariant tubular neighborhood in $M$ (cf. [Br, Theorem 2.2, p. 306]), there exists a nonconstant function $f$ in $\mathscr{C}_{G}^{\infty}(F)$. Then the dimension of $\mathscr{C}_{G}^{\infty}(F)$ is infinite. Each element $f$ in $\mathscr{C}_{G}^{\infty}(F)$ can be extended to a function $\tilde{f}$ in the space $\mathscr{C}_{v}^{\infty}(M)$ of all elements in $\mathscr{C}^{\infty}(M)$ which are invariant under horizontal transport. Since parallel transport is an isometry, the vertical Laplacian $\Delta_{v}$ leaves $\mathscr{C}_{v}^{\infty}(M)$ invariant. Therefore there exist an infinite number of eigenvalues $0 \leqslant \mu_{1} \leqslant \mu_{2} \leqslant \cdots \leqslant \mu_{i} \leqslant \cdots$, of $\Delta_{v}$ counted with their multiplicities such that

$$
-\Delta_{v} f_{i}=\mu_{i} f_{i}, \quad 0 \neq f_{i} \in \mathscr{C}_{v}^{\infty}(M), i=1,2, \ldots
$$

Now suppose that $\operatorname{Index}\left(\mathrm{id}_{N}\right)>0$, that is, there exists a nonzero element $W^{\prime}$ in $\Gamma(T N)$ such that $J_{\mathrm{id}_{N}} W^{\prime}=\lambda W^{\prime}$ and $\lambda<0$. By Proposition 7.2, (6.3), $f_{i} \in \mathscr{C}_{v}^{\infty}(M)$, (6.5) and (7.2), we have

$$
\begin{aligned}
{ }^{t} J_{\phi}\left(\widetilde{f_{i} W^{\prime}}\right) & =\left(t^{-2} J_{\phi}^{v}+J_{\phi}^{H}\right)\left(\widetilde{f_{i} W^{\prime}}\right) \\
& =t^{-2}\left(-\Delta_{v} f_{i}\right) \widetilde{W^{\prime}}+f_{i} J_{\phi}^{H}\left(\widetilde{W^{\prime}}\right)=\left(t^{-2} \mu_{i}+\lambda\right)\left(\widetilde{f_{i} W^{\prime}}\right)
\end{aligned}
$$

That is, ${ }^{t} J_{\phi}$ has the eigenvalues $t^{-2} \mu_{i}+\lambda, i=1,2, \ldots$. When $t$ goes to infinity, the eigenvalues $t^{-2} \mu_{i}+\lambda$ tend to the eigenvalue $\lambda$. Since $\lambda<0$, for each $i=1,2, \ldots$, there exists a number $N>0$ such that $t^{-2} \mu_{i}+\lambda<0$ for $t \geqslant N$. Therefore we have the desired conclusion.

REMARK. Theorem 7.5 is a generalization of Corollary 3.3 in [Sm].

\section{Homogeneous Riemannian submersions.}

8.1. In this section, we express the Jacobi operator of homogeneous Riemannian submersions in terms of Lie algebras and calculate the spectrum of the Jacobi operator of the Hopf fibration. We retain the notation of $\S 7.3$.

Let $G$ be a compact connected Lie group, with $K$ and $H$ closed subgroups of $G$. Let $g$ be the Lie algebra of $G$ consisting of all left invariant vector fields on $G$. Let $\mathfrak{f}$ and $\mathfrak{h}$ be the subalgebras corresponding to $K$ and $H$. Put $s:=\operatorname{dim} G, m:=\operatorname{dim} G / H$, and $n:=\operatorname{dim} G / K$. We choose an $\operatorname{Ad}(G)$-invariant inner product $(\cdot, \cdot)$ on $\mathfrak{g}$, with $\mathfrak{h}_{1}$ (resp. $\left.\mathfrak{p}\right)$, the orthogonal complement of $\mathfrak{h}$ (resp. $\mathfrak{f}$ ) in $\mathfrak{f}$ (resp. $\mathfrak{g}$ ). Then 
$\mathfrak{f}=\mathfrak{h} \oplus \mathfrak{h}_{1}$ with $\operatorname{Ad}(H) \mathfrak{h}_{1}=\mathfrak{h}_{1}$, and $\mathfrak{g}=\mathfrak{f} \oplus \mathfrak{p}$ with $\operatorname{Ad}(K) \mathfrak{p}=\mathfrak{p}$. Put $\mathfrak{m}:=\mathfrak{h}_{1} \oplus$ $\mathfrak{p}$, so $\mathfrak{g}=\mathfrak{h} \oplus \mathfrak{m}$ with $\operatorname{Ad}(H) \mathfrak{m}=\mathfrak{m}$. In this section, we always assume the following:

Assumption (A). We take the inner products $(\cdot, \cdot)_{\mathfrak{h}_{1}},(\cdot, \cdot)_{\mathfrak{p}}$, and $(\cdot, \cdot)_{\mathfrak{m}}$ as the restrictions to $\mathfrak{h}_{1}, \mathfrak{p}$, and $\mathfrak{m}$, respectively, of the above $\operatorname{Ad}(G)$-invariant inner product $(\cdot, \cdot)$ on $g$.

Now we consider the Riemannian submersion $\phi ; G / H \rightarrow G / K$ admitting the Riemannian metric $g$ (resp. $h$ ) on $G / H$ (resp. $G / K$ ) corresponding to the inner product $(\cdot, \cdot)$ on $\mathfrak{m}$ (resp. $\mathfrak{p}$ ). Since the induced bundle $E:=\phi^{-1} T(G / K)$ is identified with the associate bundle $G \times{ }_{H} \mathfrak{p}$, which is the space of the equivalence classes of $(x, X) \in G \times \mathfrak{p}$ under the equivalence relation $(x h, \operatorname{Ad}(h) X) \sim(x, X)$, for $h \in H$, we can identify the space $\Gamma(E)$ of its sections with the following space.

Definition 8.1. Let $\mathscr{C}^{\infty}(G, \mathfrak{p})$ be the space of all smooth maps of $G$ into $\mathfrak{p}$. We define the subspace $\mathscr{C}_{H}^{\infty}(G, \mathfrak{p})$ of $\mathscr{C}^{\infty}(G, \mathfrak{p})$ by

$$
\mathscr{C}_{H}^{\infty}(G, \mathfrak{p}):=\left\{f \in \mathscr{C}^{\infty}(G, \mathfrak{p}) ; f(x h)=\operatorname{Ad}\left(h^{-1}\right) f(x), x \in G, h \in H\right\} .
$$

The identification $\Phi$ of $\Gamma(E)$ with $\mathscr{C}_{H}^{\infty}(G, \mathfrak{p}), \Phi ; \mathscr{C}_{H}^{\infty}(G, \mathfrak{p}) \rightarrow \Gamma(E)$, is given by

$$
\Phi(f)(x H):=\tau_{x^{*}} f(x)_{\{K\}}, \quad x \in G .
$$

Here $f(x)_{\{K\}}$ is the tangent vector of $G / K$ at the origin $\{K\}$ corresponding to $f(x) \in \mathfrak{p}$, and $\tau_{x^{*}}$ is the differential of the translation $\tau_{x} ; G / K \ni y K \mapsto x y K \in G / K$. Then it turns out that $\Phi$ is an isomorphism of $\mathscr{C}_{H}^{\infty}(G, \mathfrak{p})$ onto $\Gamma(E)$. Under the $G$-actions on $\Gamma(E)$ or $\mathscr{C}_{H}^{\infty}(G, \mathfrak{p})$ defined by

$$
\begin{aligned}
& \left(\tau_{x^{*}} V\right)_{y H}:=\tau_{x^{*}} V_{x^{-1} y H}, \quad x, y \in G, V \in \Gamma(E), \\
& \left(\tau_{x} f\right)(y):=f\left(x^{-1} y\right), \quad x, y \in G, f \in \mathscr{C}_{H}^{\infty}(G, \mathfrak{p}),
\end{aligned}
$$

$\Phi$ is a $G$-isomorphism, that is,

$$
\Phi \circ \tau_{x} f=\tau_{x^{*}} \Phi(f), \quad x \in G, f \in \mathscr{C}_{H}^{\infty}(G, \mathfrak{p}) .
$$

Note that the Jacobi operator $J_{\phi} ; \Gamma(E) \rightarrow \Gamma(E)$ is $G$-invariant, that is,

$$
J_{\phi}\left(\tau_{x^{*}} V\right)=\tau_{x^{*}}\left(J_{\phi} V\right), \quad V \in \Gamma(E) .
$$

Here we denote by $\tau_{x^{*}}$ the differential of the translation $\tau_{x}$ on $G / H$ or $G / K$ by $x \in G$. Then we have $\tau_{x^{-1}{ }_{*}} \nabla_{e_{i}} e_{i}=\nabla_{\tau_{x}^{-1} e_{i}} \tau_{x^{-1}{ }_{*}} e_{i}$, and $\tilde{\nabla}_{e_{i}} \tau_{x^{*}} V=\tau_{x_{*}} \tilde{\nabla}_{\tau_{x}^{-1}{ }_{e_{i}}} V$, for $V \in$ $\Gamma(E), x \in G$, where $\left\{e_{i}\right\}_{i=1}^{m}$ is an orthonormal local frames field on $(G / H, g)$. Because of the expression (1.4) for $J_{\phi}$, we have the $G$-invariance of $J_{\phi}$.

Furthermore we identify $\mathscr{C}_{H}^{\infty}(G, \mathfrak{p})$ with the subspace $\left(\mathscr{C}^{\infty}(g) \otimes \mathfrak{p}\right)_{H}$ of the tensor product $\mathscr{C}^{\infty}(G) \otimes \mathfrak{p}$.

Definition 8.2. $\left(\mathscr{C}^{\infty}(G) \otimes \mathfrak{p}\right)_{H}$ is by definition the subspace of $\mathscr{C}^{\infty}(G) \otimes \mathfrak{p}$ consisting of all elements $\sum_{i=1}^{l} f_{i} \otimes X_{i} \in \mathscr{C}^{\infty}(G) \otimes \mathfrak{p}$ satisfying

$$
\sum_{i=1}^{l} R_{h} f_{i} \otimes \operatorname{Ad}(h) X_{i}=\sum_{i=1}^{l} f_{i} \otimes X_{i}
$$

for all $h \in H$. Here $\left(R_{h} f\right)(x):=f(x h), h \in H, x \in G, f \in \mathscr{C}^{\infty}(G)$. Under the $G$-action of $\mathscr{C}^{\infty}(G) \otimes \mathfrak{p}$ defined by

$$
\tau_{x}(f \otimes X):=\tau_{x} f \otimes X, \quad x, y \in G, f \in \mathscr{C}^{\infty}(G), X \in \mathfrak{p},
$$


the subspace $\left(\mathscr{C}^{\infty}(G) \otimes \mathfrak{p}\right)_{H}$ is a $G$-submodule. The identification $\Psi$ of $\mathscr{C}_{H}^{\infty}(G, \mathfrak{p})$ with $\left(\mathscr{C}^{\infty}(G) \otimes \mathfrak{p}\right)_{H}$ is given by

$$
\Psi(f):=\sum_{i=1}^{n} f_{i} \otimes X_{i}, \quad f \in \mathscr{C}_{H}^{\infty}(G, \mathfrak{p}),
$$

where $f(x)=\sum_{i=1}^{n} f_{i}(x) X_{i}, x \in G$, and $\left\{X_{i}\right\}_{i=1}^{n}$ is a fixed orthonormal basis of $\mathfrak{p}$ with respect to $(\cdot, \cdot)$. Then it turns out that $\Psi$ is a $G$-isomorphism of $\mathscr{C}_{H}^{\infty}(G, \mathfrak{p})$ onto $\left(\mathscr{C}^{\infty}(G) \otimes \mathfrak{p}\right)_{H}$ with

$$
\Psi \circ \tau_{x}=\tau_{x} \circ \Psi, \quad x \in G .
$$

Definition 8.3. Via $\Phi$ and $\Psi$, we can define a $G$-invariant operator $\tilde{J}$ on $\left(\mathscr{C}^{\infty}(G) \otimes \mathfrak{p}\right)_{H}$ from the Jacobi operator $J_{\phi}$ in such a way that the following diagram is commutative:

$$
\begin{array}{ccccc}
\Gamma(E) & \stackrel{\Phi^{-1}}{\rightarrow} & \mathscr{C}_{H}^{\infty}(G, \mathfrak{p}) & \stackrel{\Psi}{\rightarrow} & \left(\mathscr{C}^{\infty}(G) \otimes \mathfrak{p}\right)_{H} \\
\downarrow J_{\phi} & & & & \downarrow \tilde{J} \\
\Gamma(E) & \stackrel{\Phi^{-1}}{\rightarrow} & \mathscr{C}_{H}^{\infty}(G, \mathfrak{p}) & \stackrel{\Psi}{\rightarrow} & \left(\mathscr{C}^{\infty}(G) \otimes \mathfrak{p}\right)_{H}
\end{array}
$$

By (8.2), (8.3) and (8.5), the operator $\tilde{J}$ is $G$-invariant, that is,

$$
\tilde{J} \circ \tau_{x}=\tau_{x} \circ \tilde{J}, \quad x \in G .
$$

Therefore the problem of determining the spectrum of $J_{\phi}$ is reduced to doing so for the operator $\tilde{J}$ on $\left(\mathscr{C}^{\infty}(G) \otimes \mathfrak{p}\right)_{H}$. Thus the main purpose of this section is to express the operator $\tilde{J}$ in terms of the Lie algebra $g$ of $G$ (cf. Theorem 8.11).

8.2. For the calculus, we take a neighborhood $U$ in $G$ and a subset $N$ (resp. $N_{K}$ ) of $G$ (resp. $K$ ) in such a way that

(i) $N=U \cap \exp (\mathfrak{p}), N_{K}=U \cap \exp \left(\mathfrak{h}_{1}\right)$,

(ii) the map $N \times N_{K} \ni(y, k) \mapsto y k \in N \cdot N_{K}$ is a diffeomorphism,

(iii) the projection $\pi_{K}$ of $G$ onto $G / K$ is a diffeomorphism of $N$ onto a neighborhood $\pi_{K}(N)$ of the origin $\{K\}$ in $G / K$, and

(iv) the projection $\pi_{H}$ of $G$ onto $G / H$ is a diffeomorphism of $N \cdot N_{K}$ onto a neighborhood $\pi_{H}\left(N \cdot N_{K}\right)$ of the origin $\{H\}$ in $G / H$, where $N \cdot N_{K}:=\{y k ; y \in N$, $\left.k \in N_{K}\right\}$.

Now for an element $X \in \mathfrak{m}=\mathfrak{h}_{1} \oplus \mathfrak{p}$, define a vector field $X^{*}$ on the neighborhood $\pi_{H}\left(N \cdot N_{K}\right)$ of $\{H\}$ in $G / H$ by

$$
X_{x H}^{*}:=\tau_{x_{*}} X_{\{H\}} \in T_{x H} G / H, \quad x \in N \cdot N_{K} .
$$

Similarly, for an element $X \in \mathfrak{p}$, define a vector field $\bar{X}$ on the neighborhood $\pi_{K}(N)$ of $\{K\}$ in $G / K$ by

$$
\bar{X}_{y K}:=\tau_{y^{*}} X_{\{K\}} \in T_{y K} G / K, \quad y \in N .
$$

Let $\left\{X_{i}\right\}_{i=1}^{m}$ be an orthonormal basis of $(\mathfrak{m},(\cdot, \cdot))$ such that $\left\{X_{i}\right\}_{i=1}^{m}$ (resp. $\left.\left\{X_{i}\right\}_{i=n+1}^{m}\right)$ is a basis of $\mathfrak{p}\left(\right.$ resp. $\left.\mathfrak{h}_{1}\right)$. Then $\left\{X_{i}^{*}\right\}_{i=1}^{m}$ is an orthonormal frame field on $\pi_{H}\left(N \cdot N_{K}\right)$ such that the $X_{i}^{*}, n+1 \leqslant i \leqslant m$, are vertical and the $X_{i}^{*}, 1 \leqslant i \leqslant n$, are horizontal. Also, $\left\{\bar{X}_{i}\right\}_{i=1}^{n}$ is an orthonormal frame field on $\pi_{K}(N)$. 
REMARK. In general, the $X_{i}^{*}, 1 \leqslant i \leqslant n$, are not necessarily basic vector fields.

For every $f \in \mathscr{C}_{H}^{\infty}(G, \mathfrak{p})$, we can express $V=\Phi(f) \in \Gamma(E)$ as

$$
V_{x H}=\sum_{i=1}^{n} f_{i}(x) \tau_{x^{*}} X_{i\{K\}}, \quad x \in G,
$$

where $f(x)=\sum_{i=1}^{n} f_{i}(x) X_{i}, x \in G$. Moreover, putting

$$
\begin{gathered}
\operatorname{Ad}(k) X_{i}=\sum_{j=1}^{n} a_{i j}(k) X_{j}, \quad k \in K, \\
\tilde{f}_{j}(y k H):=\sum_{i=1}^{n} f_{i}(y k) a_{i j}(k), \quad y \in N, k \in N_{K},
\end{gathered}
$$

the section $V$ can be expressed on the neighborhood $\pi_{H}\left(N \cdot N_{K}\right)$ as

$$
V=\sum_{j=1}^{n} \tilde{f}_{j} \widetilde{\bar{X}}_{j}
$$

where $\tilde{f}_{j}$ is a function $(8.10)$ on $\pi_{H}\left(N \cdot N_{K}\right)$ and $\widetilde{\bar{X}}_{j}$ is a local section of $E$ corresponding to the vector field $\bar{X}_{j}$ on $\pi_{H}\left(N \cdot N_{K}\right)$ (cf. 1.1). Then we have for $X \in \mathfrak{m}$,

$$
\tilde{\nabla}_{X^{*}} V=\sum_{j=1}^{n}\left\{\left(X^{*} \tilde{f}_{j}\right) \widetilde{\bar{X}}_{j}+\tilde{f}_{j} \tilde{\nabla}_{X^{*}} \widetilde{\bar{X}}_{j}\right\}
$$

on $\pi_{H}\left(N \cdot N_{K}\right)$. Here $\left(\tilde{\nabla}_{X^{*}} \widetilde{\bar{X}}_{j}\right)_{x H}, x \in N \cdot N_{K}$, is given by

$$
\left(\tilde{\nabla}_{X^{*}} \widetilde{\widetilde{X}}_{j}\right)_{x H}=\left({ }^{N} \nabla_{W} \bar{X}_{j}\right)_{x K},
$$

where $W$ is a locally defined vector field on $G / K$ satisfying $W_{x K}=\phi_{*} X_{x H}^{*}$ (cf. (1.1) or (6.4)), and ${ }^{N} \nabla$ is the Levi-Civita connection of $(G / K, g)$. This vector field $W$ can be chosen as follows:

$$
\begin{gathered}
W=0 \quad \text { for } X \in \mathfrak{h}_{1}, \\
W=\frac{(\operatorname{Ad}(k(\cdot)) X)}{(\operatorname{cf} . ~(8.8)),} \text { for } X \in \mathfrak{p} .
\end{gathered}
$$

In fact, since $\phi_{*} X_{x H}^{*}=0$ for $X \in \mathfrak{h}_{1}$, we have (8.14). For (8.15), let $X \in \mathfrak{p}$. For a fixed point $x=y(x) k(x), y(x) \in N, k(x) \in N_{K}$, we have

$$
\begin{aligned}
\phi_{*} X_{x H}^{*} & =\tau_{y(x)^{*}} \tau_{k(x)^{*}} X_{\{K\}}=\tau_{y(x)_{*}}(\operatorname{Ad}(k(x)) X)_{\{K\}} \\
& ={\overline{(\operatorname{Ad}(k(x)) X)_{y(x) K}}},
\end{aligned}
$$

so we can choose $W$ as in (8.15). By (8.14), we get, for $X \in \mathfrak{h}_{1}$,

$$
\left(\tilde{\nabla}_{X^{*}} V\right)_{x H}=\sum_{j=1}^{n}\left(X^{*} \tilde{f}_{j}\right)(x H)\left(\widetilde{\widetilde{X}}_{j}\right)_{x H} .
$$

By (8.15), we get in particular, for $X \in \mathfrak{p}$,

$$
\left(\widetilde{N}^{N} \nabla_{W} \bar{X}_{j}\right)_{\{H\}}=\left({ }^{N} \nabla_{\bar{X}} \bar{X}_{j}\right)_{\{K\}} .
$$


Moreover, for $X \in \mathfrak{p}$, we will show that

$$
\left(\tilde{\nabla}_{X^{*}} \tilde{\nabla}_{X^{*}} \widetilde{\bar{X}}_{j}\right)_{\{H\}}=\frac{1}{4}\left(\left[X,\left[X, X_{j}\right]_{\mathfrak{p}}\right]_{\mathfrak{p}}\right)_{\{K\}} \in T_{\{K\}} G / K,
$$

where $X_{\mathfrak{p}}$ is the $\mathfrak{p}$-component of $X$ corresponding to the decomposition $\mathfrak{g}=\mathfrak{t}+\mathfrak{p}$.

First, recall the lemma below which follows from Theorems 8.1, 10.1 and 13.2 in [No], due to assumption (A).

LEMMA 8.4. For every $Y, Z \in \mathfrak{p}$,

$$
{ }^{N} \nabla_{\bar{Z}} \bar{Y}=\frac{1}{2} \overline{\left([Z, Y]_{\mathfrak{p}}\right)} \text {, along the curve } \xi(t) K \text { in } G / K
$$

for a sufficiently small $t$ such that $\xi(t):=\exp (t Z)$ belongs to $N$.

To establish (8.18), note that by (8.13), we have

$$
\left(\tilde{\nabla}_{X^{*}} \tilde{\nabla}_{X^{*}} \widetilde{\bar{X}}_{j}\right)_{\{H\}}=\left({ }^{N} \nabla_{W}{ }^{N} \nabla_{W} \bar{X}_{j}\right)_{\{K\}},
$$

where $W$ is given by (8.15). Then for the curve $\sigma(t):=\exp (t X) K$ in $G / K$,

$$
\text { the right side of (8.19) }=\left.\frac{d}{d t}{ }^{N} P_{\sigma(t)}^{-1}\left({ }^{N} \nabla_{W} \bar{X}_{j}\right)_{\sigma(t)}\right|_{t=0},
$$

where ${ }^{N} P_{\sigma(t)}$ is parallel transport of $(G / K, g)$ along the curve $\sigma(t)$. Here $W_{\cdot \sigma(t)}=$ $\bar{X}_{\sigma(t)}$, by $(8.15)$ and $\exp (t X) \in N$, so that $k(\sigma(t))=e$. Then we have

$$
\left({ }^{N} \nabla_{W} \bar{X}_{j}\right)_{\sigma(t)}=\left({ }^{N} \nabla_{\bar{X}} \bar{X}_{j}\right)_{\sigma(t)}=\frac{1}{2} \overline{\left(\left[X, X_{j}\right]_{\mathfrak{p}}\right)_{\sigma(t)}},
$$

by Lemma 8.4 , which also gives

$$
\text { the right side of } \begin{aligned}
(8.19) & =\frac{1}{2} \frac{d}{d t}{ }^{N} P_{\sigma(t)}^{-1} \overline{\left(\left[X, X_{j}\right]_{\mathfrak{p}}\right)} \\
& =\frac{1}{2}\left(\left.{ }^{N} \nabla_{\bar{X}(t)}\right|_{t=0} \overline{\left[X, X_{j}\right]_{\mathfrak{p}}}\right)_{\{K\}}=\frac{1}{4}\left(\left[X,\left[X, X_{j}\right]_{\mathfrak{p}}\right]_{\mathfrak{p}}\right)_{\{K\}},
\end{aligned}
$$

which implies (8.18).

Summing up the above, we have

LEMMA 8.5. For $V=\Phi(f), f \in \mathscr{C}_{H}^{\infty}(G, \mathfrak{p})$, we have

(i)

$$
\left(\tilde{\nabla}_{X^{*}} \tilde{\nabla}_{X^{*}} V\right)_{\{H\}}=\sum_{i=1}^{n} X_{\{H\}}^{*}\left(X^{*} \tilde{f}_{j}\right) \bar{X}_{j\{K\}}, \quad \text { for } X \in \mathfrak{h}_{1},
$$

(ii)

$$
\begin{aligned}
\left(\tilde{\nabla}_{X^{*}} \tilde{\nabla}_{X^{*}} V\right)_{\{H\}}= & \sum_{j=1}^{n} X_{\{H\}}^{*}\left(X^{*} \tilde{f}_{j}\right) \bar{X}_{j\{K\}}+\left(X_{\{H\}}^{*} \tilde{f}_{j}\right){\overline{\left(\left[X, X_{j}\right]_{\mathfrak{p}}\right)}}_{\{K\}} \\
& +\frac{1}{4} \tilde{f}_{j}(H) \overline{\left(\left[X,\left[X, X_{j}\right]_{\mathfrak{p}}\right]_{\mathfrak{p}}\right)_{\{K\}}}
\end{aligned}
$$

for $X \in \mathfrak{p}$. 
Our next task is to calculate $X_{\{H\}}^{*} \tilde{f}_{j}$ and $X_{\{H\}}^{*} X^{*} \tilde{f}_{j}$, for $X \in \mathfrak{m}$.

LEMma 8.6. (i) For $X \in \mathfrak{h}_{1}$, we have

$$
X_{\{H\}}^{*} \tilde{f}_{j}=X f_{j}(e)+\sum_{i=1}^{n} f_{i}(e)\left(\left[X, X_{i}\right], X_{j}\right),
$$

and

$$
\begin{aligned}
X_{\{H\}}^{*} X^{*} \tilde{f}_{j}= & X^{2} f_{j}(e)+2 \sum_{i=1}^{n}\left(X f_{i}\right)(e)\left(\left[X, X_{i}\right], X_{j}\right) \\
& +\sum_{i=1}^{n} f_{i}(e)\left(\left[X,\left[X, X_{i}\right]\right], X_{j}\right) .
\end{aligned}
$$

(ii) For $X \in \mathfrak{p}$, we have

$$
X_{\{H\}}^{*} \tilde{f}_{j}=X f_{j}(e),
$$

and

$$
X_{\{H\}}^{*} X^{*} \tilde{f}_{j}=X^{2} f_{j}(e) .
$$

Proof follows immediately from the definition of $\tilde{f}_{j}(8.9),(8.10)$ and $X^{*}(8.7)$.

LEMMA 8.7. $\left(\tilde{\nabla}_{\nabla_{X^{*}} X^{*}} V\right)_{H}=0$ for all $X \in \mathfrak{m}$, and $V \in \Gamma(E)$.

Proof. Due to assumption (A), we have $\left(\nabla_{X^{*}} X^{*}\right)_{\{H\}}=0$ for $X \in \mathfrak{m}$, by Theorems 8.1, 13.1 in [No]. By (8.13) or (1.1), we have Lemma 8.7.

Moreover, it is known (cf. [K.N]) that under assumption (A), the curvature tensor ${ }^{N} R$ of $(G / K, h)$ is given by

$$
\begin{aligned}
-\left({ }^{N} R(X, Y) Z\right)_{\{K\}}= & \frac{1}{4}\left[X,[Y, Z]_{\mathfrak{p}}\right]_{\mathfrak{p}}-\frac{1}{4}\left[Y,[X, Z]_{\mathfrak{p}}\right]_{\mathfrak{p}}-\frac{1}{2}\left[[X, Y]_{\mathfrak{p}}, Z\right]_{\mathfrak{p}} \\
& -\left[[X, Y]_{\mathfrak{f}}, Z\right], \quad X, Y, Z \in \mathfrak{p},
\end{aligned}
$$

where we identify $X \in \mathfrak{p}$ with the tangent vector $X_{\{K\}} \in T_{\{K\}} G / K$. Then we get

LeMma 8.8. For $V=\Phi(f), f \in \mathscr{C}_{H}^{\infty}(G, \mathfrak{p})$, we have

$$
\begin{aligned}
-\left({ }^{N} R\left(\phi_{*} X^{*}, V\right) \phi_{*} X^{*}\right)_{\{K\}} & \\
\quad= & \left\{\begin{aligned}
0, \quad X \in \mathfrak{h}_{1}, \\
\sum_{i=1}^{n} f_{i}(e)\left\{\frac{1}{4}\left[X,\left[X_{i}, X\right]_{\mathfrak{p}}\right]_{\mathfrak{p}}\right. \\
\left.-\frac{1}{2}\left[\left[X, X_{i}\right]_{\mathfrak{p}}, X\right]_{\mathfrak{p}}-\left[\left[X, X_{i}\right]_{\mathfrak{f}}, X\right]\right\}, \quad X \in \mathfrak{P} .
\end{aligned}\right.
\end{aligned}
$$


Summing up Lemmas 8.5-8.8, we obtain

Proposition 8.9. For $V=\Phi(f)$ and $f=\sum_{i=1}^{n} f_{i} X_{i} \in \mathscr{C}_{H}^{\infty}(G, \mathfrak{p})$, the evaluation of $J_{\phi} V$ at the origin $\{H\}$ in $G / H$ is given by

$$
\begin{aligned}
\left(J_{\phi} V\right)_{\{H\}}= & -\sum_{k=1}^{m} \sum_{j=1}^{n}\left(X_{k}^{2} f_{j}\right)(e) X_{j\{K\}} \\
& -\sum_{k, j=1}^{n}\left(X_{k} f_{j}\right)(e)\left[X_{k}, X_{j}\right]_{\mathfrak{p}\{K\}} \\
& -2 \sum_{k=n+1}^{m} \sum_{j=1}^{n}\left(X_{k} f_{j}\right)(e)\left[X_{k}, X_{j}\right]_{\{K\}} \\
& -\sum_{k=n+1}^{m} \sum_{j=1}^{n} f_{j}(e)\left[X_{k},\left[X_{k}, X_{j}\right]\right]_{\{K\}} \\
& -\sum_{k, j=1}^{n} f_{j}(e)\left[\left[X_{k}, X_{j}\right]_{\mathfrak{k}}, X_{k}\right]_{\{K\}} .
\end{aligned}
$$

8.3. Before we state Theorem 8.11 , we need some notation.

Definition 8.10. The operators $D_{i}, i=0,1, \ldots, 6$, acting on $\mathscr{C}^{\infty}(G) \otimes \mathfrak{p}$ are given by

$$
\begin{aligned}
& D_{0}:=\sum_{k=1}^{s} X_{k}^{2} \otimes I, \\
& D_{1}:=\sum_{k=1}^{m} X_{k}^{2} \otimes I, \\
& D_{2}:=\sum_{k=1}^{n} X_{k} \otimes P_{\mathfrak{p}} \circ \operatorname{ad}\left(X_{k}\right), \\
& D_{3}:=\sum_{k=n+1}^{m} X_{k} \otimes \operatorname{ad}\left(X_{k}\right), \\
& D_{4}:=I \otimes \sum_{k=n+1}^{m} \operatorname{ad}\left(X_{k}\right)^{2}, \\
& D_{5}:=I \otimes \sum_{k=1}^{n} \operatorname{ad}\left(X_{k}\right) \circ P_{\mathfrak{f}} \circ \operatorname{ad}\left(X_{k}\right), \\
& D_{6}:=\sum_{k=m+1}^{s} X_{k}^{2} \otimes I,
\end{aligned}
$$

where $P_{\mathfrak{p}}$ and $P_{\mathfrak{t}}$ are the projections of $\mathfrak{g}=\mathfrak{f} \oplus \mathfrak{p}$ onto $\mathfrak{p}$ and $\mathfrak{f}$, respectively, $\left\{X_{k}\right\}_{k=1}^{s}$ is an orthonormal basis of $(\mathrm{g},(\cdot, \cdot))$ such that $\left\{X_{i}\right\}_{i=1}^{n}$ (resp. $\left\{X_{i}\right\}_{i=n+1}^{m}$, $\left\{X_{i}\right\}_{i=m+1}^{s}$ ) is a basis of $\mathfrak{p}\left(\right.$ resp. $\left.\mathfrak{h}_{1}, \mathfrak{h}\right), I$ is the identity operator of $\mathscr{C}^{\infty}(G), \mathfrak{p}$ or $\mathscr{C}^{\infty}(G) \otimes \mathfrak{p}$, and $(X f)(x):=\left.(d / d t) f(x \exp (t X))\right|_{t=0}$, for $X \in \mathfrak{g}, f \in \mathscr{C}^{\infty}(G)$, and $x \in G$. 
It turns out that all $D_{i}, i=0,1, \ldots, 6$, are independent of the choice of the above basis $\left\{X_{k}\right\}_{k=1}^{s}$ and they are $G$-invariant, i.e., $D_{i} \circ \tau_{x}=\tau_{x} \circ D_{i}$, for all $x \in G$. Thus since $R_{h} \circ X f=\operatorname{Ad}(h) X\left(R_{h} f\right)$, for $f \in \mathscr{C}^{\infty}(G), h \in H$, and $X \in \mathfrak{g}$, all $D_{i}$ keep the subspace $\left(\mathscr{C}^{\infty}(G) \otimes \mathfrak{p}\right)_{H}$ invariant. We also note that

$$
D_{0}=D_{1}+D_{6} \text {, }
$$

$$
D_{6}=I \otimes \sum_{k=m+1}^{s} \operatorname{ad}\left(X_{k}\right)^{2} \text { on }\left(\mathscr{C}^{\infty}(G) \otimes \mathfrak{p}\right)_{H},
$$

by the definition of $\left(\mathscr{C}^{\infty}(G) \otimes \mathfrak{p}\right)_{H}$ and $D_{6}$. Then by Proposition 8.9 , we obtain

THEOREM 8.11. Let $\phi$ be the Riemannian submersion of $(G / H, g)$ onto $(G / K, h)$ whose metrics $g$ and $h$ come from the $\operatorname{Ad}(G)$-invariant inner product $(\cdot, \cdot)$ on the Lie algebra $\mathrm{g}$. Then the operator $\tilde{J}$ of $\left(\mathscr{C}^{\infty}(G) \otimes \mathfrak{p}\right)_{H}$ corresponding to the Jacobi operator $J_{\phi}$ of the submersion $\phi$ coincides with the operator

$$
D:=-D_{0}-D_{2}-2 D_{3}-D_{4}+D_{5}+D_{6},
$$

where all $D_{i}$ are defined in Definition 8.10.

Proof. Proposition 8.9 and (8.21) yield

$$
\tilde{J}\left(\Psi \Phi^{-1} V\right)(e)=D\left(\Psi \Phi^{-1} V\right)(e),
$$

for every $V \in \Gamma(E)$. For every $x \in G$, we have

$$
\begin{aligned}
& \tilde{J}\left(\Psi \Phi^{-1} V\right)(x)=\tau_{x^{-1}} \circ \tilde{J}\left(\Psi \Phi^{-1} V\right)(e)=\tilde{J}\left(\Psi \Phi^{-1} \tau_{x^{-1 *}} V\right)(e) \\
& =D\left(\Psi \Phi^{-1} \tau_{x^{-1 *}} V\right)(e)=\tau_{x^{-1}} D\left(\Psi \Phi^{-1} V\right)(e)=D\left(\Psi \Phi^{-1} V\right)(e) \text {. Q.E.D. }
\end{aligned}
$$

As applications of Theorem 8.11, we obtain

COROllary 8.12. Let $\phi$ be the Riemannian submersion of $(G / H, g)$ onto $(G / K, h)$ whose metrics $g$ and $h$ come from the $\operatorname{Ad}(G)$-invariant inner product $(\cdot, \cdot)$ on the Lie algebra g. Assume that $(G / K, h)$ is Riemannian symmetric, $g$ is semisimple, and $(X, Y):=-F(X, Y)$, for $X, Y \in \mathrm{g}$, where $F$ is the Killing form of $\mathrm{g}$.

(i) Then the operator $\tilde{J}$ of $\left(\mathscr{C}^{\infty}(G) \otimes \mathfrak{p}\right)_{H}$ corresponding to the Jacobi operator $J_{\phi}$ of the submersion $\phi$ coincides with

$$
D:=-D_{0}-2 D_{3}+2 D_{6} \text {. }
$$

If $H=\{\mathrm{id}\}$, then the operator $\tilde{J}$ coincides with $D:=-D_{0}-2 D_{3}$, where $D_{0}, D_{3}$ and $D_{6}$ are given in Definition 8.10 .

(ii) In particular, the spectrum of the Jacobi operator $J_{\phi}$ of the Hopf fibering $\phi$;

$$
(S U(2), g)=\left(S^{3}, g\right) \rightarrow(S U(2) / S(U(1) \times U(1)), h)=\left(S^{2}, h\right)
$$

is given as follows: The eigenvalues: $\frac{1}{2} l(l+1)+i, \frac{1}{2} l(l+1)-i$, their multiplicities: $2 l+1$, where $l$ varies over the set $\left\{l \in \frac{1}{2} \mathbf{Z} ; l \geqslant 0\right\}$, and $i$ varies over the set $\{l, l-1, \ldots, 1-l,-l\}$. Finally, $\operatorname{Index}(\phi)=2$ and $\operatorname{Nullity}(\phi)=8$.

Proof. (i) Since $(G / K, h)$ is symmetric, i.e., $[\mathfrak{p}, \mathfrak{p}] \subset \mathfrak{f}$, we have $D_{2}=0$ and $D_{5}=I \otimes \sum_{k=1}^{n} \operatorname{ad}\left(X_{k}\right)^{2}$. Moreover, $D_{5}=-\frac{1}{2} I$ and $D_{4}+D_{6}=-\frac{1}{2} I$ imply (i), since $\left(\sum_{k=1}^{n} \operatorname{ad}\left(X_{k}\right)^{2}(X), Y\right)=\frac{1}{2} F(X, Y)$, and $\left(\sum_{k=n+1}^{s} \operatorname{ad}\left(X_{k}\right)^{2}(X), Y\right)=\frac{1}{2} F(X, Y)$, for $X, Y \in \mathfrak{p}$ (cf. [T.K, p. 212]). The second claim in (i) is clear because $D_{6}=0$ when $H=\{$ id $\}$. 
(ii) Let us recall the computation in $[\mathbf{U 1}, \S 5]$. In this case,

$$
\begin{aligned}
& G=S U(2), \\
& K=S(U(1) \times U(1))=\left\{\left(\begin{array}{cc}
e^{\sqrt{-1} \theta} & 0 \\
0 & e^{-\sqrt{-1} \theta}
\end{array}\right) ; \theta \in \mathbf{R}\right\}, \\
& (X, Y)=-4 \operatorname{Trace}(X Y), \quad X, Y \in \mathfrak{g}=\mathfrak{g} \mathfrak{H}(2), \\
& \mathfrak{f}=\left\{H_{1}\right\}_{\mathbf{R}}, \\
& \mathfrak{p}=\left\{U_{\alpha} / \sqrt{2}, V_{\alpha} / \sqrt{2}\right\}_{\mathbf{R}},
\end{aligned}
$$

where

$$
H_{1}:=\sqrt{-1} / 2 \sqrt{2}\left(\begin{array}{cc}
1 & 0 \\
0 & -1
\end{array}\right), \quad U_{\alpha}:=2^{-1}\left(\begin{array}{cc}
0 & \sqrt{-1} \\
\sqrt{-1} & 0
\end{array}\right)
$$

and

$$
V_{\alpha}:=2^{-1}\left(\begin{array}{ll}
0 & 1 \\
1 & 0
\end{array}\right)
$$

Here $\left\{H_{1}, U_{\alpha} / \sqrt{2}, V_{\alpha} / \sqrt{2}\right\}$ is an orthonormal basis of $(g,(\cdot, \cdot))$. We have only to know the actions of $D_{3}=H_{1} \otimes \operatorname{ad}\left(H_{1}\right)$ and $D_{0}=C \otimes I$ on $\mathscr{C}^{\infty}(G) \otimes \mathfrak{p}$, where $C$ is the Casimir operator $C:=H_{1}^{2}+U_{\alpha}^{2} / 2+V_{\alpha}^{2} / 2$. A complete orthogonal basis of the space $\mathscr{C}_{\mathbf{C}}^{\infty}(G)$ of complex valued smooth functions on $G$ with respect to the inner product $\int_{G} f(x) f^{\prime}(x) d x, f, f^{\prime} \in \mathscr{C}_{\mathbf{C}}^{\infty}(g)$, with the Haar measure $d x$, is given as follows by the Peter-Weyl theorem. Let $D:=\left\{l \alpha ; l \in \frac{1}{2} \mathbf{Z}, l \geqslant 0\right\}$. For $\lambda=l \alpha \in D$, let $\left(V_{\lambda}, \pi^{\lambda}\right)$ be the irreducible unitary representation of $G$ with highest weight $\lambda$, and $\left\{v_{i}\right\}_{i=1}^{d_{\lambda}}, d_{\lambda}:=\operatorname{dim}\left(V_{\lambda}\right)$, an orthonormal basis of $V_{\lambda}$ with respect to the $G$-invariant inner product $((\cdot, \cdot))$ on $V_{\lambda}$. Put $\pi_{i j}^{\lambda}(x):=\left(\left(\pi^{\lambda}(x) v_{i}, v_{j}\right)\right), 1 \leqslant i, j \leqslant d_{\lambda}$. Then

$$
X \pi_{i j}^{\lambda}(x)=\left(\left(\pi^{\lambda}(x) \pi^{\lambda}(X) v_{i}, v_{j}\right)\right), \quad X \in g, 1 \leqslant i, j \leqslant d_{\lambda},
$$

and $\left\{\pi_{i j}^{\lambda}, \lambda \in D, 1 \leqslant i, j \leqslant d_{\lambda}\right\}$ is an orthogonal basis of $\mathscr{C}_{\mathbf{C}}^{\infty}(G)$. For $\lambda=l \alpha$ with $l \in \frac{1}{2} \mathbf{Z}, l \geqslant 0, V_{\lambda}$ has an orthonormal basis $\left\{v_{m} ; m=l, l-1, \ldots, 1-l,-l\right\}$ such that

$$
\pi^{\lambda}\left(H_{1}\right) v_{m}=\sqrt{-1} m v_{m} / \sqrt{2}
$$

for each $m$. Since $\pi^{\lambda}(C)=\frac{1}{2} l(l+1) I$ on $V_{\lambda}$, we get

$$
\begin{gathered}
H_{1} \pi_{i j}^{\lambda}(x)=(\sqrt{-1} / \sqrt{2}) i \pi_{i j}^{\lambda}(x), \\
C \pi_{i j}^{\lambda}(x)=\frac{1}{2} l(l+1) \pi_{i j}^{\lambda}(x),
\end{gathered}
$$

for $i, j=l, l-1, \ldots, 1-l,-l$. On the other hand,

$$
\operatorname{ad}\left(H_{1}\right)\left(\frac{U_{\alpha}}{\sqrt{2}}\right)=\frac{1}{\sqrt{2}}\left(\frac{V_{\alpha}}{\sqrt{2}}\right), \quad \operatorname{ad}\left(H_{1}\right)\left(\frac{V_{\alpha}}{\sqrt{2}}\right)=-\frac{1}{\sqrt{2}}\left(\frac{U_{\alpha}}{\sqrt{2}}\right)
$$


Thus the action of $D_{3}=H_{1} \otimes \operatorname{ad}\left(H_{1}\right)$ on $\vartheta_{\lambda} \otimes \mathfrak{p}$, where $\vartheta_{\lambda}:=\left\{\pi_{i j}^{\lambda} ; 1 \leqslant i, j \leqslant d_{\lambda}\right\}_{\mathbf{C}}$, is equivalent to the matrix

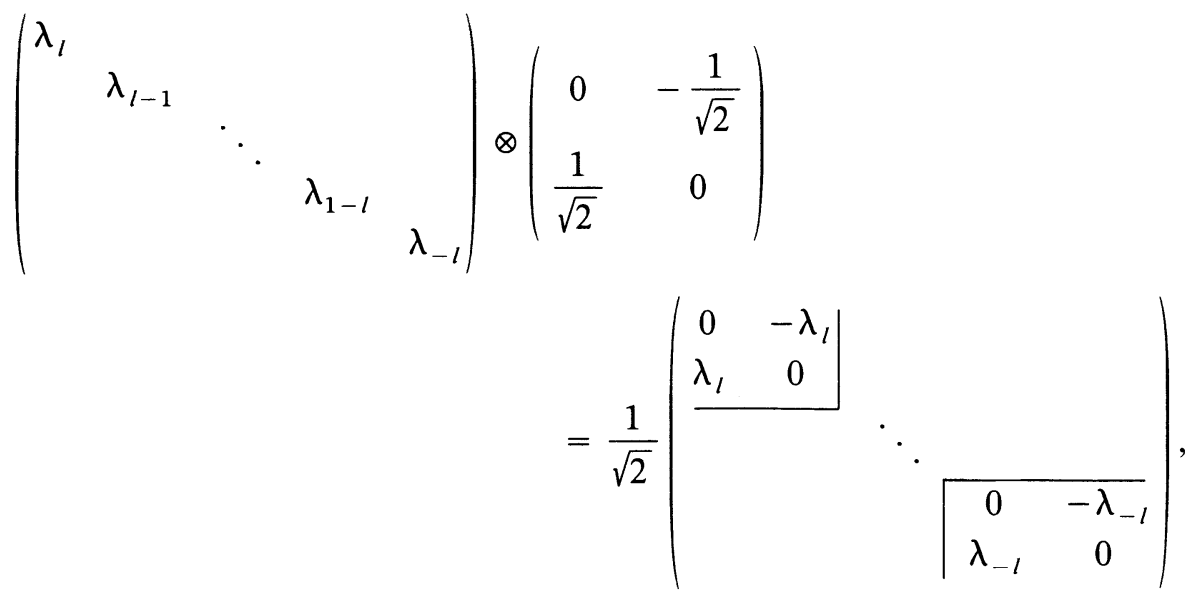

where $\lambda_{i}:=(\sqrt{-1} / \sqrt{2}) i, i=l, l-1, \ldots, 1-l,-l$. Therefore the eigenvalues of $D_{3}$ on $\vartheta_{\lambda} \otimes \mathfrak{p}$ are given by $\pm \frac{i}{2}, i=l, l-1, \ldots, 1-l,-l$. Hence the spectrum of $D=-D_{0}-2 D_{3}$ is given as in (ii). Q.E.D.

Instead of the assumption of Corollary 8.12, we now assume that $K=H$. In this case, we obtain the formula for $\tilde{J}$ of the Jacobi operator $J_{\text {id }}$ of the identity map of a normally homogeneous space $(G / H, g)$. Here we have $\mathfrak{f}=\mathfrak{h}, \mathfrak{h}_{1}=0, \mathfrak{m}=\mathfrak{p}$ and $D_{3}=D_{4}=0$. Thus we obtain

Corollary 8.13. Let $(G / H, g)$ be a normally homogeneous space, that is, the metric $g$ is induced from the $\operatorname{Ad}(G)$-invariant inner product $(\cdot, \cdot)$ on the Lie algebra $\mathrm{g}$. Then the operator $\tilde{J}$ of $\left(\mathscr{C}^{\infty}(G) \otimes \mathfrak{m}\right)_{H}$ corresponding to the Jacobi operator $J_{\text {id }}$ of the identity map of $(G / H, g)$ coincides with $D=-D_{0}-D_{2}+D_{5}+D_{6}$, where $\mathrm{m}$ is the orthogonal complement of $\mathfrak{h}$ in $\mathfrak{g}$ with respect to $(\cdot, \cdot)$ and $D_{0}, D_{2}, D_{5}$ and $D_{6}$ are given in Definition 8.10.

In particular, assume that $(G / H, g)$ is Riemannian symmetric, $g$ is semisimple, and $(X, Y):=-F(X, Y)$ for $X, Y \in \mathfrak{g}$, where $F$ is the Killing form of $\mathfrak{g}$. Then $D=-D_{0}-I$, where $I$ is the identity map of $\left(\mathscr{C}^{\infty}(G) \otimes \mathfrak{m}\right)_{H}$.

Proof. The last formula follows from $D_{2}=0$ and $D_{5}+D_{6}=-I$.

REMARK. The last formula $D=-D_{0}-I$ for the Jacobi operator of the identity map of a Riemannian symmetric space was stated in [Na].

\section{REFERENCES}

[B.G] P. Béard and S. Gallot, Inégalités isopérimétriques pour l'équation de la chaleur et application a l'estimation de quelques invariants, Séminaire Goulaouic-Meyer-Schwartz, 1983-1984, No. 15, 1983.

[B.B] L. Bérard Bergery and J. P. Bourguignon, Laplacians and Riemannian submersions with totally geodesic fibers, Illinois J. Math. 26 (1982), 181-200.

[B.G.M] M. Berger, P. Gauduchon and E. Mazet, Le spectre d' une variété riemannienne, Lecture Notes in Math., vol. 194, Springer-Verlag, Berlin and New York, 1871.

[B.O] R. L. Bishop and B. O'Neill, Manifolds of negative curvature, Trans. Amer. Math. Soc. 145 (1969), 1-49. 
[B.L] J. P. Bourguignon and B. Lawson, Stability and isolation phenomena for Yang-Mills fields, Comm. Math. Phys. 79 (1981), 189-230.

[B.M] P. Bérard and D. Meyer, Inégalités isopérimétriques et applications, Ann. Sci. Ecole Norm. Sup. 15 (1982), 513-542.

[Br] G. R. Bredon, Introduction to compact transformation groups, Academic Press, New York, 1972.

[C.L] S. Y. Cheng and P. Li, Seminar on differential geometry, (S. T. Yau, ed.), Ann. of Math. Studies, no. 102, Princeton, N. J., 1982, p. 22.

[E.L] J. Eells and L. Lemaire, A report on harmonic maps, Bull. London Math. Soc. 10 (1978), 1-68.

[E.S] J. Eells and J. H. Sampson, Harmonic mappings of Riemannian manifolds, Amer. J. Math. 86 (1964), 109-160.

[F] G. B. Folland, Introduction to partial differential equations, Mathematical Notes, Princeton Univ. Press, Princeton, N. J., 1976.

[G.K.M] D. Gromoll, W. Klingenberg and W. Meyer, Riemannsche Geometrie im Großen, Lecture Notes in Math., vol. 55, Springer, New York, 1975.

[H.S.U] H. Hess, R. Schrader and D. A. Uhlenbrock, Kato's inequality and the spectral distribution of Laplacians on compact Riemannian manifolds, J. Differential Geom. 15 (1980), 27-38.

[H] D. Hoffman, Lower bounds on the first eigenvalue of the Laplacian of Riemannian submanifolds; Minimal submanifolds and geodesics, Kaigai, Tokyo, 1978, pp. 61-73.

[J] G. R. Jensen, Einstein metrics on principal fiber bundles, J. Differential Geom. 8 (1973), 599-614.

[K.N] S. Kobayashi and K. Nomizu, Foundations of differential geometry. II, Interscience, New York, 1969.

[L] A. Lichnérowicz, Applications harmoniques et variétés Kählerienne, Sympos. Math. III, Bologna, 1970, pp. 341-402.

[Ma] E. Mazet, La formule de la variation seconde de l'énergie au voisinage d'une application harmonique, J. Differential Geom. 8 (1973), 279-296.

[Mo] H. Mori, Notes on the stability of minimal submanifolds of Riemannian manifolds, Yokohama Math. J. 25 (1977), 9-15.

[M.U] H. Muto and H. Urakawa, On the least positive eigenvalue of the Laplacian on compact homogeneous spaces, Osaka J. Math. 17 (1980), 471-484.

[Na] T. Nagano, Stability of harmonic maps between symmetric spaces, Harmonic maps, Proc. Tulane, Lecture Notes in Math., vol. 949, Springer-Verlag, Berlin and New York, 1982, pp. 130-137.

[No] K. Nomizu, Invariant affine connections on homogeneous spaces, Amer. J. Math. 76 (1954), 33-65.

[Ob] M. Obata, Riemannian manifolds admitting a solution of a certain system of differential equations, Proc. U.S.-Japan Semin. J. Differential Geom., Kyoto, Japan, 1965, pp. 101-114.

[O.N] B. O'Neill, The fundamental equations of a submersion, Michigan Math. J. 13 (1966), 459-469.

[Si] J. Simons, Minimal varieties in Riemannian manifolds, Ann. of Math. (2) 88 (1968), 62-105.

[Sm] R. T. Smith, The second variation formula for harmonic mappings, Proc. Amer. Math. Soc. 47 (1975), 229-236.

[T] M. Takeuchi, Modern theory of spherical functions, Iwanami, Tokyo, 1975. (Japanese)

[T.K] M. Takeuchi and S. Kobayashi, Minimal imbeddings of R-spaces, J. Differential Geom. 2 (1968), 203-215.

[T1] S. Tanno, Geometric expressions of eigen 1-forms of the Laplacian on spheres; Spectra of Riemannian manifolds, Kaigai, Tokyo, 1983, pp. 115-128.

[T2] __ Remarks on Sobolev inequalities and stability of minimal submanifolds, J. Math. Soc. Japan 35 (1983), 323-329.

[U1] H. Urakawa, On the least positive eigenvalue of the Laplacian for compact group manifolds, J. Math. Soc. Japan 31 (1979), 209-226.

[U2] _ Lower bounds for the eigenvalues of the fixed vibrating membrane problems, Tôhoku Math. J. 36 (1984), 185-189.

[W] J. A. Wolf, Spaces of constant curvature, McGraw-Hill, New York, 1967.

[X] Y. L. Xin, Some results on stable harmonic maps, Duke Math. J. 47 (1980), 609-613.

[Y.B] K. Yano and S. Bochner, Curvature and Betti numbers, Ann. of Math. Studies, no. 32, Princeton Univ. Press, Princeton, N. J., 1953.

Department of Mathematics, College of General Education, Tôhoku University, Kawauchi, SENDAI, 980, JAPAN 